\title{
Relation between photospheric magnetic field and chromospheric emission
}

\author{
R. Rezaei ${ }^{1}$, R. Schlichenmaier ${ }^{1}$, C. A. R. Beck ${ }^{1,2}$, J. H. M. J. Bruls ${ }^{1}$, and W. Schmidt ${ }^{1}$ \\ 1 Kiepenheuer-Institut für Sonnenphysik, Schöneckstr. 6, 79104 Freiburg, Germany \\ e-mail: rrezaei@kis.uni-freiburg.de \\ 2 Instituto de Astrofísica de Canarias (IAC), 38205 La Laguna, Espain
}

Received 22 December 2006 / Accepted 25 January 2007

\section{ABSTRACT}

\begin{abstract}
Aims. We investigate the relationship between the photospheric magnetic field and the emission of the mid chromosphere of the Sun. Methods. We simultaneously observed the Stokes parameters of the photospheric iron line pair at $630.2 \mathrm{~nm}$ and the intensity profile of the chromospheric $\mathrm{Ca}$ II $\mathrm{H}$ line at $396.8 \mathrm{~nm}$ in a quiet Sun region at a heliocentric angle of $53^{\circ}$. Various line parameters have been deduced from the $\mathrm{Ca}$ II $\mathrm{H}$ line profile. The photospheric magnetic field vector has been reconstructed from an inversion of the measured Stokes profiles. After alignment of the $\mathrm{Ca}$ and Fe maps, a common mask has been created to define network and inter-network regions. We perform a statistical analysis of network and inter-network properties. The $H$-index is the integrated emission in a $0.1 \mathrm{~nm}$ band around the Ca core. We separate a non-magnetically, $H_{\text {non }}$, and a magnetically, $H_{\text {mag }}$, heated component from a non-heated component, $H_{\text {co }}$ in the $H$-index.

Results. The average network and inter-network $H$-indices are equal to 12 and $10 \mathrm{pm}$, respectively. The emission in the network is correlated with the magnetic flux density, approaching a value of $H \approx 10 \mathrm{pm}$ for vanishing flux. The inter-network magnetic field is dominated by weak field strengths with values down to $200 \mathrm{G}$ and has a mean absolute flux density of about $11 \mathrm{Mx} \mathrm{cm}^{-2}$.

Conclusions. We find that a dominant fraction of the calcium emission caused by the heated atmosphere in the magnetic network has non-magnetic origin $\left(H_{\mathrm{mag}} \approx 2 \mathrm{pm}, H_{\mathrm{non}} \approx 3 \mathrm{pm}\right)$. Considering the effect of straylight, the contribution from an atmosphere with no temperature rise to the $H$-index $\left(H_{\mathrm{co}} \approx 6 \mathrm{pm}\right)$ is about half of the observed $H$-index in the inter-network. The $H$-index in the inter-network is not correlated to any property of the photospheric magnetic field, suggesting that magnetic flux concentrations have a negligible role in the chromospheric heating in this region. The height range of the thermal coupling between the photosphere and low/mid chromosphere increases in presence of magnetic field. In addition, we demonstrate that a poor signal-to-noise level in the Stokes profiles leads to a significant over-estimation of the magnetic field strength.
\end{abstract}

Key words. Sun: photosphere - Sun: chromosphere - Sun: magnetic fields

\section{Introduction}

The dominant pattern covering the entire solar surface, except sunspots, is granulation, the top of small-scale convection cells with diameters of 1-2 Mm. On a larger scale of $\sim 20 \mathrm{Mm}$ supergranules are observed which show the same pattern as granulation: a horizontal flow from the center towards the boundary of the cell. These large scale convection cells have a mean lifetime of $20 \mathrm{~h}$, much longer than the granular time-scale (some $10 \mathrm{~min}$ ). The chromospheric network forms at the boundary of the supergranular cells. The network is presumably formed by the longtime advection of magnetic flux to the boundaries of the supergranules (Priest et al. 2002; Cattaneo et al. 2003). The interior of the network cells, the inter-network, has much less magnetic field than the network (e.g., Keller et al. 1994). The magnetic and thermodynamic properties of the network and inter-network are different (e.g., Lites 2002).

The chromospheric heating mechanism is one of the main challenges of solar physics (Narain \& Ulmschneider 1996, and references therein). The core emission of the $\mathrm{Ca}$ II $\mathrm{H}$ and $\mathrm{K}$ lines is an important source of radiative losses in the chromosphere. Moreover, this emission is an important tool to study the temperature stratification and the magnetic activity of the outer atmosphere of the Sun and other stars (e.g., Schrijver \& Zwaan 2000). Most of the observational studies based on these lines use either the calcium intensity profile (e.g., Cram \& Damé 1983; Lites et al. 1993) or combinations of calcium filtergrams and magnetograms (e.g., Berger \& Title 2001). From the observation of the calcium spectrum alone, it is not possible to distinguish between the magnetic and non-magnetic heating components. Combining calcium filtergrams with magnetograms allows to separate those components, but the spectral information is lost. Simultaneous observations that allow to reconstruct the magnetic field and record the spectrum for the $\mathrm{Ca}$ II $\mathrm{H}$ line are rare (e.g., Lites et al. 1999) and only available at lower spatial resolution. Therefore, it is not surprising that none of the present theories, mechanical and Joule heating, was confirmed or rejected observationally (Fossum \& Carlsson 2005; Socas-Navarro 2005).

Schrijver (1987) studied a sample of late-type stars and introduced the concept of the basal flux to separate the non-magnetic heating from the magnetic one. The Sun is not a very active star, with a chromospheric radiative loss on the order of the basal flux for Sun-like stars (Fawzy et al. 2002b). Carlsson \& Stein (1997) presented a semi-empirical hydrodynamic model where enhanced chromospheric emission is due to outward propagating acoustic waves. This model was criticized in subsequent investigations (e.g., Kalkofen et al. 1999; Fossum \& Carlsson 2005), which was partly due to disagreements on the temperature stratification in higher layers (e.g., Ayres 2002; WedemeyerBöhm et al. 2005). In addition, it is not clear whether high- or 

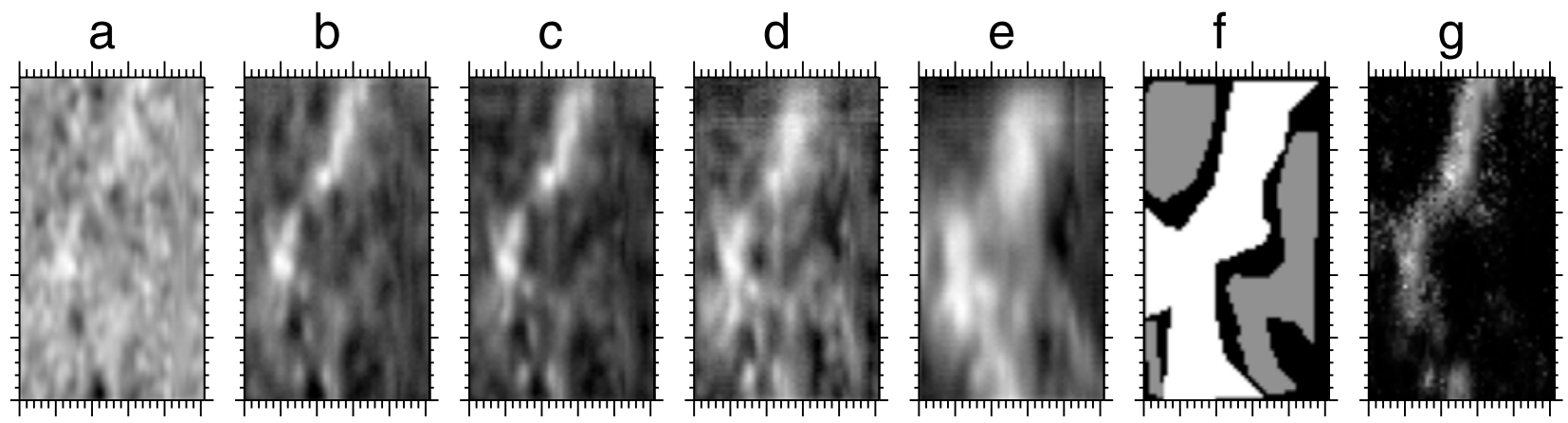

Fig. 1. From left to right: a) the Fe I $630 \mathrm{~nm}$ continuum intensity, b) the calcium wing intensity at $396.490 \mathrm{~nm}$ which was calibrated to FTS data (Stenflo et al. 1984), c) the outer calcium wing intensity $(W 1)$, d) the inner calcium wing intensity (W3), e) the $H$-index, f) the masks which separate network (white) from the inter-network (gray), and g) the magnetic flux density obtained from the inversion. We did not use the black region between the network and inter-network. Each small tickmark is 1 arcsec. Note that sampling in $x$ and $y$ directions are different.

low-frequency acoustic waves play the dominant role in the energy transport to higher layers (Fawzy et al. 2002a; Jefferies et al. 2006). While there are theoretical indications that the magnetic filling factor discriminates between different regimes of heating (Solanki \& Steiner 1990), there is no canonic model reproducing thermal, dynamical and magnetic properties of the solar chromosphere (Judge \& Peter 1998; Rutten 1999).

The POlarimetric LIttrow Spectrograph (POLIS, Schmidt et al. 2003; Beck et al. 2005a) was designed to provide cotemporal and co-spatial measurements of the magnetic field in the photosphere and the Ca II H intensity profile. We use POLIS to address the question of the chromospheric heating mechanism, by comparing properties of network and inter-network in photosphere and chromosphere by a statistical analysis. With the information on the photospheric fields, we separate the contributions of the magnetically and non-magnetically heated component. For the first time we study the correlation of the chromospheric emission with the corresponding amplitude/area asymmetry and Stokes- $V$ zero-crossing velocity at the corresponding photospheric position. We also investigate the magnetic field strength distribution of the inter-network to check whether it consists of weak fields (e.g., Faurobert et al. 2001; Collados 2001) or kilo-Gauss fields (e.g., Sánchez Almeida et al. 2003a; Sánchez Almeida et al. 2003b).

Observations and data reduction are explained in Sects. 2 and 3. Histograms of the obtained parameters are presented in Sect. 4. Correlations between the chromospheric and photospheric parameters are addressed in Sect. 5. The heating contributions are elaborated in Sect. 6. Discussion and conclusions are presented in Sects. 7 and 8, respectively. Details of the magnetic field parameters in the inter-network are discussed in Appendix A. The straylight contamination and calibration uncertainties for the POLIS Ca channel are estimated in Appendix B. An overview of some of our results also appears in Rezaei et al. (2007).

\section{Observations}

We observed a network area close to the active region NOAA 10675 on September 27, 2004, with the POLIS instrument at the German Vacuum Tower Telescope (VTT) in Tenerife. The observations were part of a coordinated observation campaign in the International Time Program, where also the Swedish Solar Telescope (SST), Dutch Open Telescope (DOT) and the Télescope Héliographique pour l'Étude du Magnétisme et des Instabilités Solaires (THÉMIS) participated. Here we

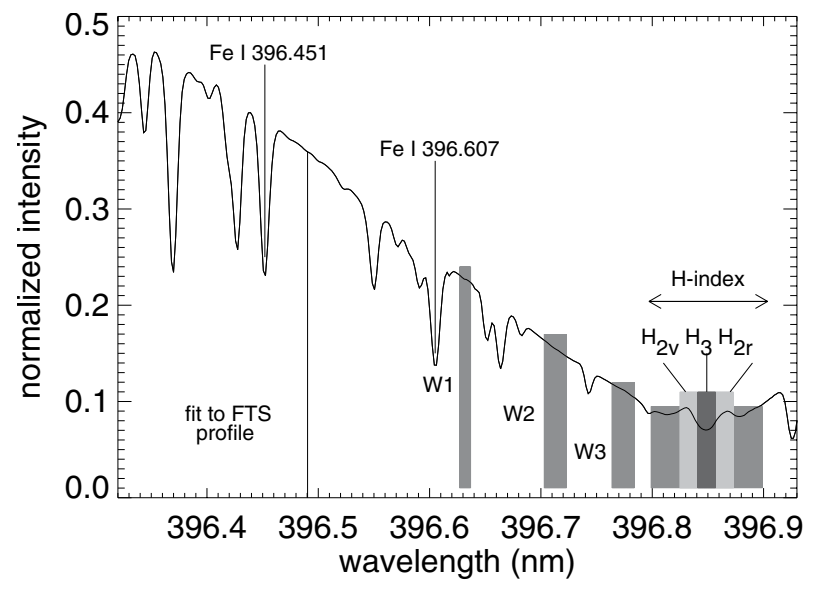

Fig. 2. Sample averaged calcium profile of one of the maps (the average profile is similar for all other maps). The bands are explained in Table 1.

analyze a series of thirteen maps of the same network region at a heliocentric angle of $53^{\circ}$, taken during several hours. We achieved a spatial resolution of around 1.0 arcsec, estimated from the spatial power spectrum of the intensity maps.

All maps were recorded with a slit width of 0.5 arcsec, a slit height of 47.5 arcsec, and an exposure time per slit position of $4.92 \mathrm{~s}$. The scan extension for the first three maps was 40.5, 55.5, and 20.5 arcsec, while it was 25.5 arcsec for the remaining ten maps.

The $\mathrm{CaIIH}$ line and the visible neutral iron lines at $630.15 \mathrm{~nm}, 630.25 \mathrm{~nm}$, and Ti I $630.38 \mathrm{~nm}$ were observed with the blue $(396.8 \mathrm{~nm})$ and red $(630 \mathrm{~nm})$ channels of POLIS. The spatial sampling along the slit ( $y$-axis in Fig. 1) was 0.29 arcsec. The scanning step was 0.5 arcsec for all maps. The spectral sampling of $1.92 \mathrm{pm}$ for the blue channel and $1.49 \mathrm{pm}$ for the red channel leads to a velocity dispersion of 1.45 and $0.7 \mathrm{~km} \mathrm{~s}^{-1}$ per pixel, respectively. The spectropolarimetric data of the red channel were corrected for instrumental effects and telescope polarization with the procedures described by Beck et al. (2005a,b).

Figure 1 displays an overview of one of the thirteen maps after spatial alignment. The map (a) shows the Fe I $630 \mathrm{~nm}$ continuum intensity normalized to the average quiet Sun intensity. The map (b) shows the $\mathrm{Ca}$ II $\mathrm{H}$ wing intensity, taken close to $396.490 \mathrm{~nm}$ (for a definition of line parameters see Fig. 2 and Table 1). These two maps were used for the spatial alignment of the red and blue channels. The next two maps (c and d) show the intensities in the outer and inner wings ( $W 1$, 
Table 1. The definition of the characteristic parameters of the $\mathrm{Ca}$ II $\mathrm{H}$ profile for the peak sample (upper part) and the band sample (lower part) (see also Fig. 2). Wavelengths are in $\mathrm{nm}$.

\begin{tabular}{cc}
\hline \hline quantity: peak sample & description \\
\hline$H_{3}$ & core intensity \\
$H_{2 \mathrm{v}}$ & violet emission peak \\
$H_{2 \mathrm{r}}$ & red emission peak \\
$V / R$ & $H_{2 \mathrm{v}} / H_{2 \mathrm{r}}$ \\
emission strength & $H_{2 \mathrm{v}} / H_{3}$ \\
$\lambda\left(H_{3}\right)$ & calcium core wavelength \\
$\lambda\left(H_{2 \mathrm{v}}\right)$ & $H_{2 \mathrm{v}}$ wavelength \\
$\lambda\left(H_{2 \mathrm{r}}\right)$ & $H_{2 \mathrm{r}}$ wavelength \\
\hline quantity: band sample & description \\
\hline$H$-index & $396.849 \pm 0.050$ \\
$H_{3}$ & $396.849 \pm 0.008$ \\
$H_{2 \mathrm{v}}$ & $396.833 \pm 0.008$ \\
$H_{2 \mathrm{r}}$ & $396.865 \pm 0.008$ \\
$W 1$ & outer wing: $396.632 \pm 0.005$ \\
$W 2$ & middle wing: $396.713 \pm 0.010$ \\
$W 3$ & inner wing: $396.774 \pm 0.010$ \\
\hline
\end{tabular}

respectively, $W 3$ ). The inner wing samples a wavelength band close to the core; hence, it is more influenced by the line-core emission and shows higher contrast of the network than the outer wing. The next map (e) is the $H$-index, i.e., the intensity of the calcium core integrated over $0.1 \mathrm{~nm}$ (cf. Table 1). The network features appear broadest and show the highest contrast in this map. The map (f) demonstrates network and inter-network masks (Sect. 3.1). The map (g) shows the magnetic flux density obtained from the spectro-polarimetric data (Sect. 3.2).

\section{Data analysis}

In this section we discuss characteristic parameters of the calcium profile and the Fe I $630 \mathrm{~nm}$ line pair. We briefly explain the inversion method that was used to infer the vector magnetic field.

\subsection{Definition of network and inter-network}

For each map, we created a mask to distinguish between the network and inter-network regions (Fig. 1f). This was done manually on the basis of the magnetic flux and the $H$-index. We did not use the black region in Fig. If which separates network from inter-network. To study structures in these two regions, we define two statistical samples:

The peak sample: Each member of this sample has a regular $V$ profile and a double reversal in the calcium core. For this sample, we study quantities related to the $\mathrm{CaIIH}$ emission peaks (Table 1, upper part). The number of data points in the network and inter-network are 19509 and 1712, respectively. We emphasize that the few inter-network points in this sample are not distributed uniformly. They usually belong to small-scale magnetic elements surrounded by large areas without magnetic signal above the noise level.

The band sample: All network and inter-network points, marked in the masks, are present in this sample. Since some of these profiles do not have two emission peaks in the calcium core, we use integrated intensities in a fixed spectral range (Cram \& Damé 1983) at the core and wing of the calcium profile

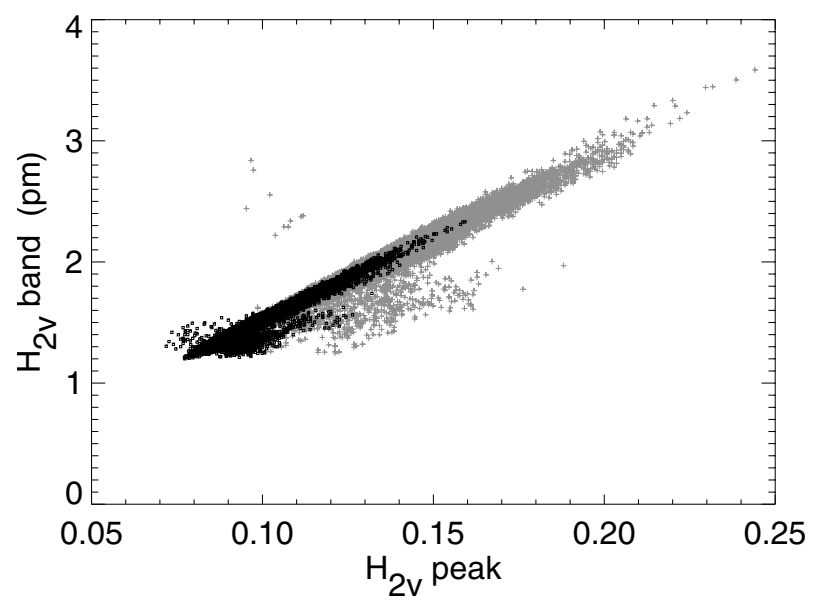

Fig. 3. Scatter plot of the $H_{2 v}$ band vs. peak definition. In the band definition, a fixed spectral range was used. Gray and black show the network and inter-network, respectively.

(Table 1, lower part). This definition retrieves reasonable chromospheric parameters. In total from all the maps, 24225 points were selected as network (white in Fig. 1f) and 20855 as internetwork positions (gray in Fig. 1f).

\subsection{Analysis of $\mathrm{Ca} I \mathrm{H}$ profiles}

We averaged profiles over a large area (including network) to obtain an average profile for each map. These average profiles are the mean of more than three thousand profiles each time; an example is shown in Fig. 2. We then normalized the intensity at the line wing at $396.490 \mathrm{~nm}$ to the FTS profile (Stenflo et al. 1984). The same normalization coefficient was applied to all profiles of the map. Hence, all profiles are normalized to the average continuum intensity.

Table 1 lists the characteristic parameters we define for each calcium profile:

a) The intensity of the $\mathrm{Ca}$ II $\mathrm{H}$ core, $H_{3}$, and the amplitudes of the emission peaks, $H_{2 \mathrm{v}}$ and $H_{2 \mathrm{r}}$, and their respective positions, $\lambda_{\mathrm{H}_{3}}, \lambda_{\mathrm{H}_{2 \mathrm{v}}}$, and $\lambda_{\mathrm{H}_{2 \mathrm{r}}}$ (peak sample).

b) Intensities in the outer (W1), middle (W2), and inner line wings (W3), which are integrated over spectral bands with a fixed spectral range (band sample).

From these quantities we derive:

1. the ratio of the emission peaks, $V / R=H_{2 \mathrm{v}} / H_{2 \mathrm{r}}$, and

2. the emission strength which is the intensity of the violet emission peak divided by the core, $\mathrm{H}_{2 \mathrm{v}} / \mathrm{H}_{3}$.

The band intensities are defined such that the wavelength intervals are fixed for all the profiles and do not depend on the peak/core positions (Table 1, lower part). Figure 3 shows a comparison between the $H_{2 \mathrm{v}}$ parameters derived from the band and peak definitions. The correlation is very strong (also for other peak parameters). Thus, it is justified to use the values from the band sample to improve the statistics for the inter-network.

\subsection{Analysis of $\mathrm{Fel} 630 \mathrm{~nm}$ line profiles}

The data of the red channel of POLIS were treated with the standard polarimetric calibration procedures described in Beck et al. $(2005 a, b)$. The spectral line curvature was removed using the telluric $\mathrm{O}_{2}$ line at $630.20 \mathrm{~nm}$ with a routine described in 

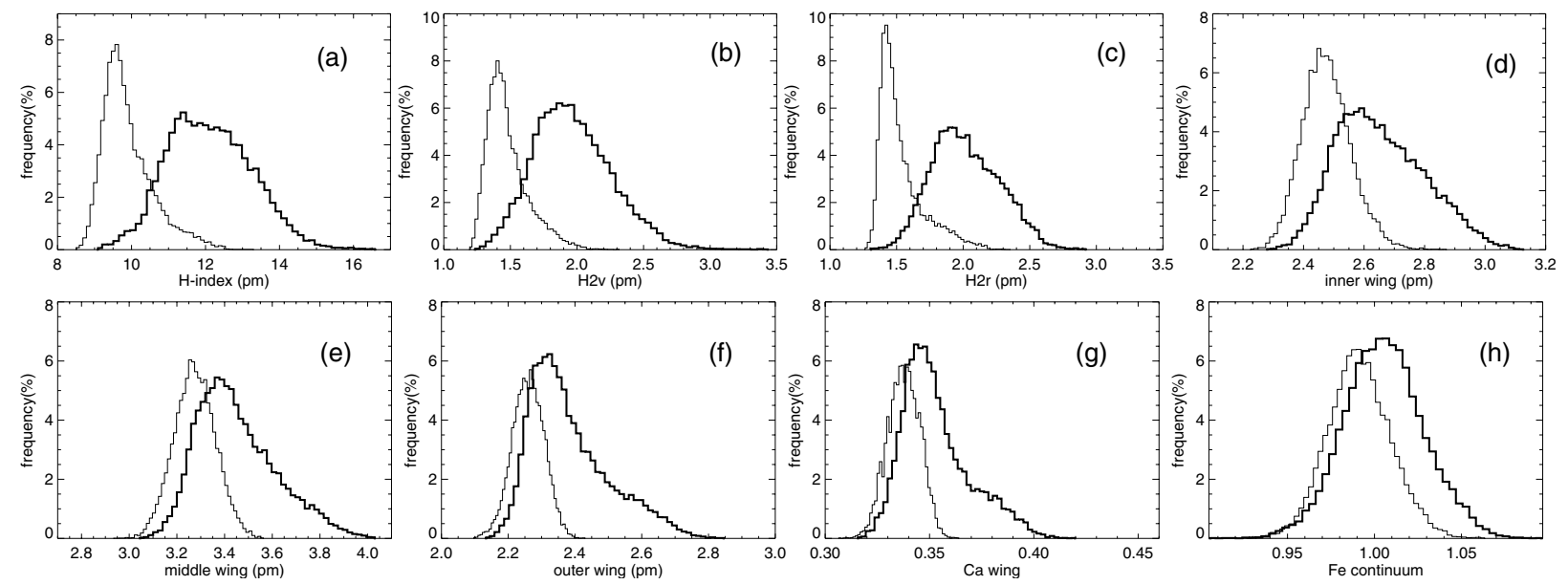

Fig. 4. Histograms of the intensity parameters for the network (thick) and inter-network (thin), using the band sample: a) the $H$-index, b) $H_{2 \mathrm{v}}$, c) $H_{2 \mathrm{r}}$, d) $W 3$ (the inner wing intensity), e) $W 2$ (the middle wing intensity), f) $W 1$ (the outer wing intensity), g) the calcium wing intensity at $396.490 \mathrm{~nm}$ which was used for normalization (see Fig. 2), and h) Fe I630.2 nm normalized continuum intensity (see Table 1 for definitions).

Rezaei et al. (2006). The polarization signal in $Q(\lambda), U(\lambda)$, and $V(\lambda)$ is normalized to the local continuum intensity, $I_{\mathrm{c}}$, for each pixel. The rms noise level of the Stokes parameters in the continuum was $\sigma=8.0 \times 10^{-4} I_{\mathrm{c}}$. Only pixels with $V$ signals greater than $3 \sigma$ were included in the profile analysis. For regular Stokes $-V$ signals above the threshold, we derived positions and amplitudes of the profile extrema in all Stokes parameters by fitting a parabola to each lobe.

An inversion was performed using the SIR code (Ruiz Cobo $\&$ del Toro Iniesta 1992). We used the same setup as in Bellot Rubio \& Beck (2005) and Beck et al. (2006): a twocomponent solar atmosphere model with one magnetic and one field-free component. Additionally, a variable amount of straylight was allowed for. This accounts for unresolved magnetic fields inside each pixel. We did not consider any gradient for the atmospheric parameters except for the temperature. The inversion yields the magnetic field vector and an estimate for the magnetic filling fraction. The flux density map (magnetic flux per pixel, Fig. $1 \mathrm{~g}$ ) is based on the inversion results.

\section{Histograms}

\subsection{Parameters of the intensity profiles}

The network patches are brighter than the inter-network at all wavelengths: in the core and wing of the $\mathrm{CaIIH}$ line and in $\mathrm{Fe}$ I $630 \mathrm{~nm}$ continuum. The distributions of the intensity parameters at different wavelengths are shown in Fig. 4. Thick and thin curves show network and inter-network, respectively. A comparison between the network and inter-network histograms indicates that the calcium core parameters $\left(H\right.$-index, $\left.H_{2 \mathrm{v}}, H_{2 \mathrm{r}}\right)$ are more shifted than the wing/continuum intensities (Figs. $4 \mathrm{a}-\mathrm{c}$ ). In other words, the intensity contrast increases from the continuum toward the wing and peaks at the core. Moreover, the wing intensities show different behaviors due to their respective distances to the core: the closer the selected wavelength band to the core, the larger the shift between network and internetwork distributions. Hence, the histogram of the inner wing intensity (Fig. 4d) has a larger shift than the middle and outer wings (Figs. $4 \mathrm{e}$ and $\mathrm{f}$ ). The distribution of the $\mathrm{Ca}$ II $\mathrm{H}$ wing ${ }^{1}$ and $\mathrm{Fe}$ I $630 \mathrm{~nm}$ continuum intensities also show a shift between

${ }^{1}$ Here, wing means the wavelength that was used for the intensity calibration (see Fig. 2). the network and inter-network: the peak of the distribution in the network is slightly brighter than in the inter-network in $396.490 \mathrm{~nm}$ and also in the $630 \mathrm{~nm}$ continuum (Figs. $4 \mathrm{~g}$ and $\mathrm{h}$ ).

\subsection{Magnetic field parameters}

From the inversion, we obtain the magnetic field and the magnetic flux in the photosphere. The statistics of these quantities in the network and inter-network are discussed here; the relation with the chromospheric parameters is discussed in the next section. We restrict the analysis to those profiles with a regular Stokes- $V$ and a double emission calcium profile, although there are only 1712 profiles in the inter-network. The histogram of the absolute magnetic flux density, $\Phi=f B \cos \alpha$, is shown in Fig. 5a, where $f$ is the magnetic filling factor, $B$ is the field strength, and $\alpha$ is the angle between the magnetic field orientation and the line-of-sight. The histogram for the inter-network has a narrow distribution, which peaks at some $\sim 4 \mathrm{Mx} \mathrm{cm}^{-2}$ whereas there is a long tail up to $\sim 250 \mathrm{Mx} \mathrm{cm}^{-2}$ for the network. The distribution function of the magnetic field strength (Fig. 5b) has a peak around $\sim 1.3 \mathrm{kG}$ for the network elements and increases with decreasing field strength for the internetwork. The peak at $200 \mathrm{G}$ is due to the detection limit of the polarization signal. The histogram of the $V$ amplitude (Fig. 5c) shows weak inter-network signals with a $V$ amplitude far less than $1 \%$. The distribution of the inter-network $V$ amplitude ${ }^{2}$ peaks at $\sim 2 \times 10^{-3} I_{\mathrm{c}}$ whereas the network shows a broad distribution up to $\sim 0.03 I_{\mathrm{c}}$.

The $V$ velocity distribution is shown in Fig. 5d. Although it has a peak around zero both for the network and inter-network, the inter-network shows a larger fraction of high-velocity $V$ profiles. The amplitude and area asymmetries in the network peak at small positive values of $10 \%$ and $3 \%$, respectively (Figs. 5e and $\mathrm{f}$ ). In contrast, there is no tendency to positive or negative values for the inter-network asymmetries. This is in agreement with other studies of quiet Sun magnetic fields (Sigwarth et al. 1999; Sigwarth 2001; Khomenko et al. 2003).

\footnotetext{
${ }^{2}$ Histogram of the $V$-amplitude of the inter-network (Fig. 5c) includes all data points with a clear $V$ signal.
} 

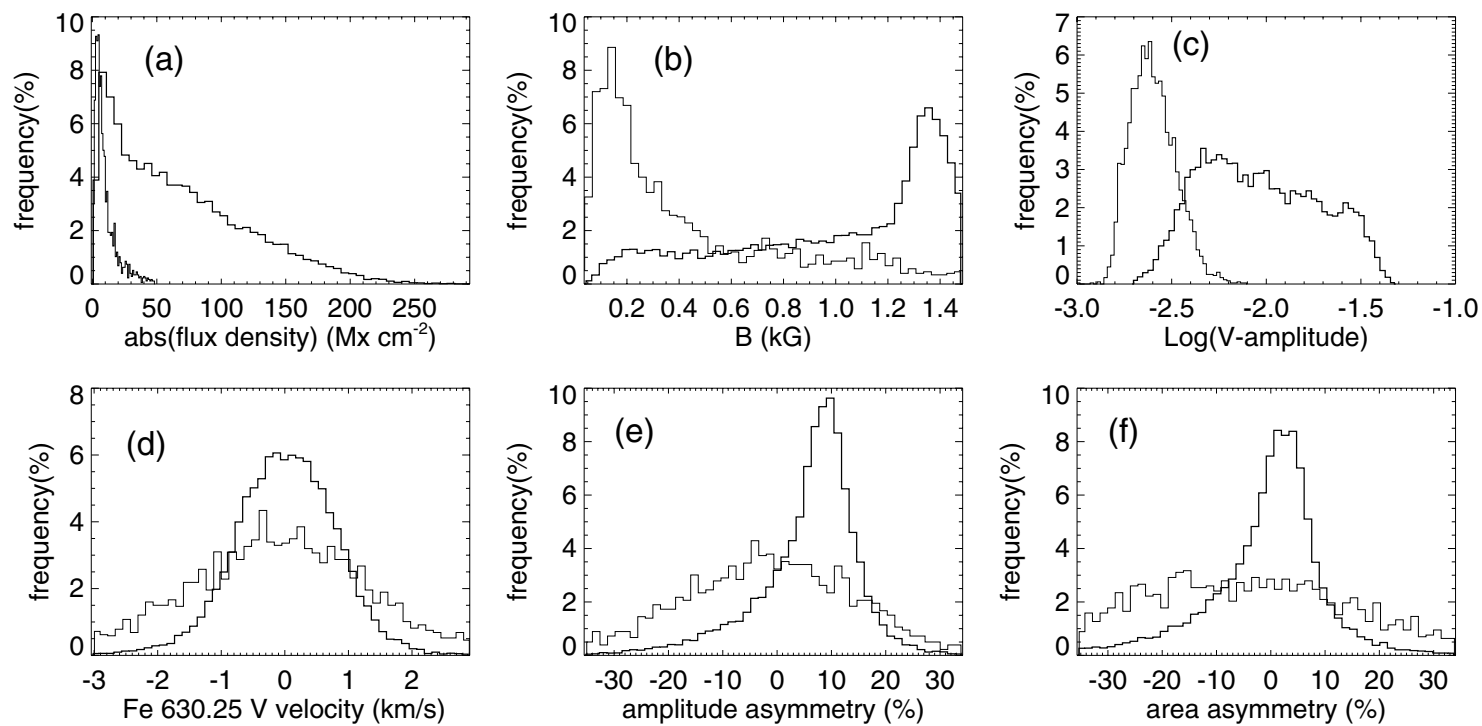

Fig. 5. Histograms of the magnetic field parameters for the network (thick) and inter-network (thin), using the peak sample: a) the absolute magnetic flux density, b) the field strength, c) Stokes $-V$ amplitude, d) Fe I $630.25 \mathrm{~nm} V$ velocity, e) the amplitude asymmetry, and f) the area asymmetry.
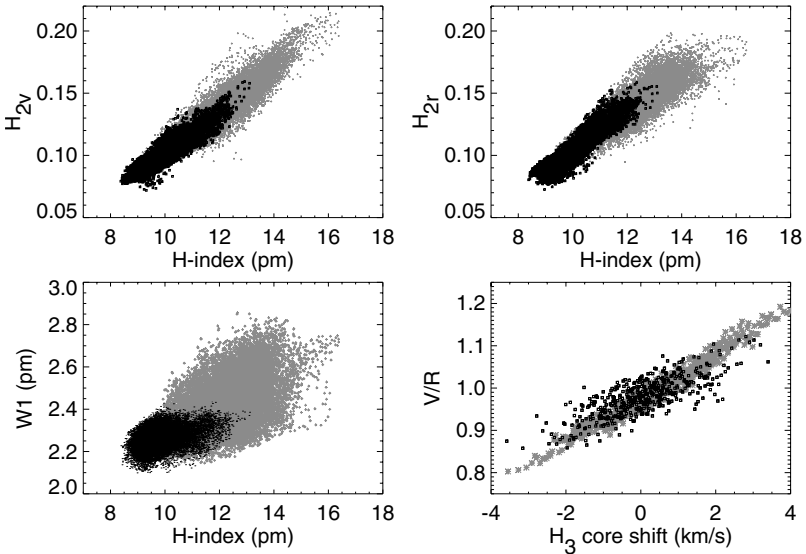

Fig. 6. Upper panels: correlation between the $H_{2 \mathrm{v}}$ and $H_{2 \mathrm{r}}$ based on the peak sample and the $H$-index. Lower left: correlation between $W_{1}$ and the $H$-index. Lower right: correlation of the $(V / R)$ ratio with the calcium core position. Gray and black show the network and inter-network, respectively (for abbreviations, see Table 1).

\section{Correlations}

\subsection{Correlations between chromospheric quantities}

There is a strong correlation between the $H$-index, $H_{3}, H_{2 \mathrm{v}}, H_{2 \mathrm{r}}$, and the inner wing intensity (with a correlation coefficient $\geq 0.8$, see Fig. 6). Therefore, we adopt the $H$-index as a proxy for the calcium core emission. It also has the advantage to be the calcium intensity parameter closest to the widely used filtergrams ${ }^{3}$.

The $(V / R)$ ratio is an indicator for the $H_{3}$ line-core position (Rutten \& Uitenbroek 1991; Cram \& Damé 1983). There is a strong correlation between the $(V / R)$ ratio and $\lambda\left(H_{3}\right)$ : the redshifted and blueshifted calcium profiles correspond to $(V / R)$ ratios smaller and larger than one, respectively (Fig. 6, lower

${ }^{3}$ Note that filtergrams, e.g., from DOT (de Wijn et al. 2005) also cover parts of the line wing while we integrate over a band with a width of $.1 \pm 0.001 \mathrm{~nm}$
Table 2. Correlation coefficients between the band intensities and the $H$-index in the network and inter-network using the band sample.

\begin{tabular}{ccc}
\hline \hline parameter & network & inter-network \\
\hline$H_{3}$ & 0.88 & 0.90 \\
$H_{2 \mathrm{v}}$ & 0.92 & 0.94 \\
$H_{2 \mathrm{r}}$ & 0.88 & 0.93 \\
$W 3$ & 0.77 & 0.68 \\
$W 2$ & 0.73 & 0.53 \\
$W 1$ & 0.58 & 0.36 \\
\hline
\end{tabular}

right panel $)^{4}$. We find a similar correlation between the emission strength and the $(V / R)$ ratio: the larger the $(V / R)$ ratio, the larger the emission strength. The lower left panel of Fig. 6 shows the correlation between the outer wing intensity and the $H$-index. While there is a correlation in the network (gray), there is no significant correlation in the inter-network (Table 2). We calculated the correlation coefficient between the $H$-index and all intensities in the line wing to investigate this difference in more detail (Table 2). In the network, there is a stronger correlation than in the inter-network for all the wing bands considered. We return to this point in Sect. 7.

\subsection{Correlations between photospheric quantities}

There is a strong correlation between the amplitude and area asymmetries, both in the network and inter-network (Fig. 7, top left panel). In contrast, there is no correlation between either amplitude or area asymmetries and the $V$ velocity (bottom left panel, Fig. 7). These are typical properties of the quiet Sun magnetic field (e.g., Sigwarth et al. 1999). The right panels of Fig. 7 show scatter plots of the $V$ asymmetries versus $V$ amplitude. There is a tendency for high amplitude $V$ signals to have small asymmetries. On the other hand, histograms of the asymmetries peak at a positive value. Implications of these two findings will be discussed in Sect. 5.3.2.

\footnotetext{
4 The calcium core position is expressed in velocity units for comparison. This does not mean that it corresponds to a Doppler shift.
} 

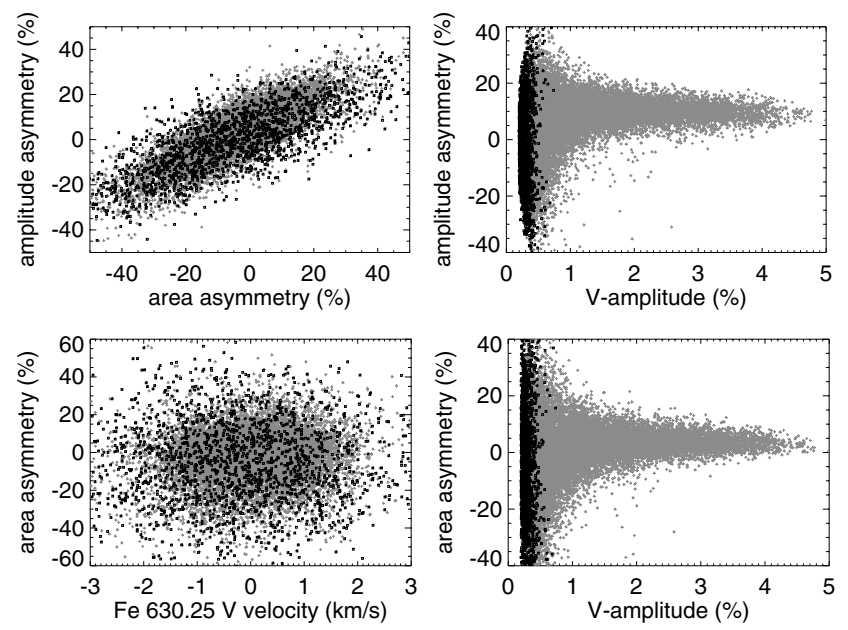

Fig. 7. Top left: scatter plot of the amplitude vs. area asymmetries. Bottom left: scatter plot of the area asymmetry vs. the $V$ velocity. Right panels: scatter plots of the amplitude and area asymmetries vs. the Fe I $630.25 \mathrm{~nm} V$ amplitude. Gray and black show the network and inter-network, respectively.

\subsection{Correlations between photospheric and chromospheric quantities}

\subsubsection{Calcium core emission vs. magnetic flux}

The upper panel of Fig. 8 shows the relation between the photospheric magnetic flux and the chromospheric emission for the network. For lower flux values $\left(<100 \mathrm{Mx} \mathrm{cm}^{-2}\right)$, there is a clear increase of emission with flux. However, for higher magnetic flux densities, the $H$-index increases slowly. To reproduce the observed relation, we utilize a power law fit to the data,

$H=a \Phi^{b}+c$,

where $\Phi$ is the absolute magnetic flux density, $H$ is the $H$-index, $a$ is a constant coefficient, $b$ is the power index, and $c$ is the non-magnetic contribution (see Sect. 5.3.2). We use a variable lower threshold of $0,3, . ., 20 \mathrm{Mx} \mathrm{cm}^{-2}$ (cf. Table 3 ) for the fit and neglect all data points with fluxes below this level. We find that the power exponent, $b$, depends strongly on the threshold. The higher the threshold, the better the fit curve resembles a straight line, as first reported by Skumanich et al. (1975). If we keep all the points, including inter-network, we obtain a value for the power exponent $b$ of $\approx 0.2$.

The calcium core emission in the inter-network does not correlate with the magnetic flux, at least with the concentrated magnetic field within the Zeeman sensitivity (Fig. 8, lower panel). The value of the offset, $c$, of the fit to the network (Table 3 ) for low magnetic flux densities is around $10 \mathrm{pm}$, which is consistent with the average $H$-index value of all the inter-network profiles ( $10 \mathrm{pm}$ for the peak sample). The value of the $c$ parameter changes for different thresholds of the flux density (Table 3 ). Implications of this finding for the basal flux are discussed in Sect. 7.

\subsubsection{Calcium core emission vs. Stokes $-V$ velocity and area asymmetry}

There are different behaviors for the network and inter-network calcium core emission with respect to $V$ velocity and asymmetries (Fig. 9). The core emission peaks at small positive amplitude/area symmetry in the network. This is caused by strong
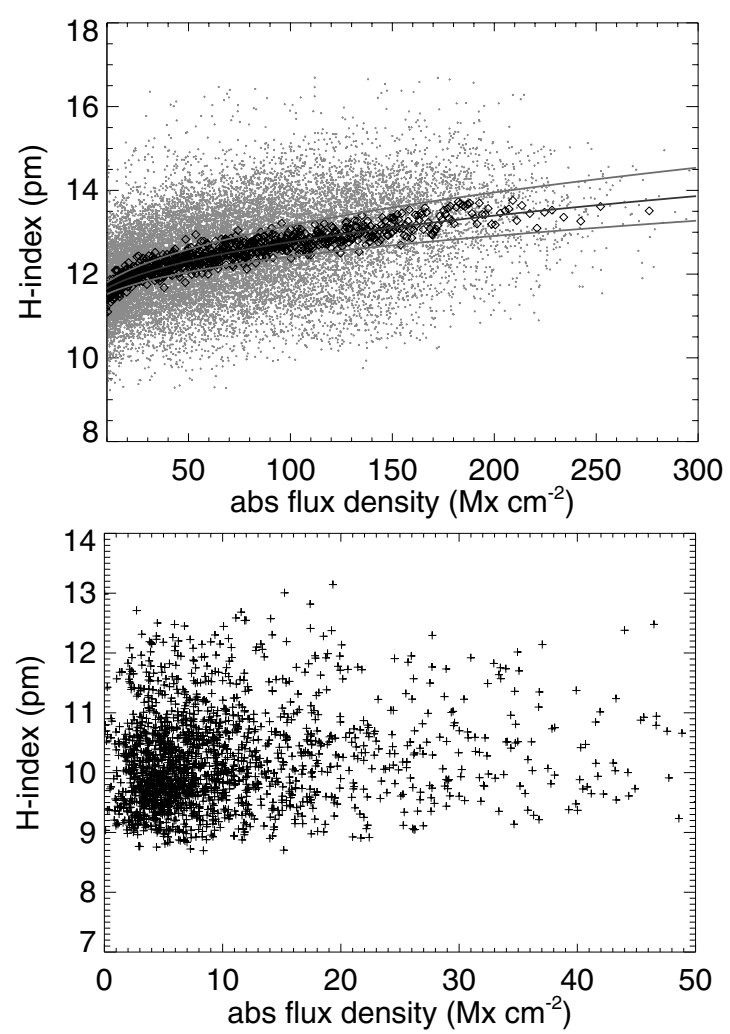

Fig. 8. Upper panel: correlation between the $H$-index and the absolute magnetic flux density. Gray is the original data and black is the binned data: each point is average of 25 points. The middle curve shows a fit of a power law to the original data (Eq. (1)), the other two curves give the $1-\sigma$ error range of the fit. Lower panel: there is no correlation between the $H$-index and the magnetic flux density in inter-network.

Table 3. The parameters of the fit to Eq. (1) to the network data. The first column is the threshold for the magnetic flux density $\left(\mathrm{Mx} \mathrm{cm}^{-2}\right)$. By increasing the threshold, we avoid the inter-network intrusions. For a comparison to the peak inter-network flux density, see Appendix A.

\begin{tabular}{cccc}
\hline \hline cut & $a(\mathrm{pm})$ & $b$ & $c(\mathrm{pm})$ \\
\hline 0 & $0.73 \pm 0.05$ & $0.28 \pm 0.01$ & $10.1 \pm 0.1$ \\
3 & $0.71 \pm 0.05$ & $0.29 \pm 0.01$ & $10.1 \pm 0.1$ \\
5 & $0.61 \pm 0.04$ & $0.31 \pm 0.01$ & $10.3 \pm 0.1$ \\
10 & $0.22 \pm 0.02$ & $0.45 \pm 0.02$ & $11.0 \pm 0.1$ \\
20 & $0.14 \pm 0.06$ & $0.51 \pm 0.06$ & $11.3 \pm 0.2$ \\
\hline
\end{tabular}

$V$ profiles that show small asymmetries (Fig. 7, right panels). The highest $H$-index in the network corresponds to almost zero $V$ velocity. In its diagram (Fig. 9), the slope of the left branch (blueshift) is smaller than the right one. Moreover for the strong upflow or downflow in the magnetic atmosphere in the network, the $H$-index decreases significantly. The $H$-index in the internetwork does not depend on any parameter of the Stokes- $V$ profile (Fig. 9).

Considering the fact that the amplitude and area asymmetries strongly correlate with each other (top left panel, Fig. 7), a natural consequence is that the left (right) branch of the scatter plot of the $H$-index vs. amplitude asymmetry corresponds to the left (right) branch of the scatter plot of the $H$-index vs. area asymmetry (Fig. 9). However, if we compare only negative or positive branches of the scatter plots of the $H$-index vs. $V$ velocity and asymmetries in the network, we realize that the left branch in the $V$ velocity does not correspond to the similar branch in the asymmetry plots. The left diagrams in Fig. 10 consider only the 

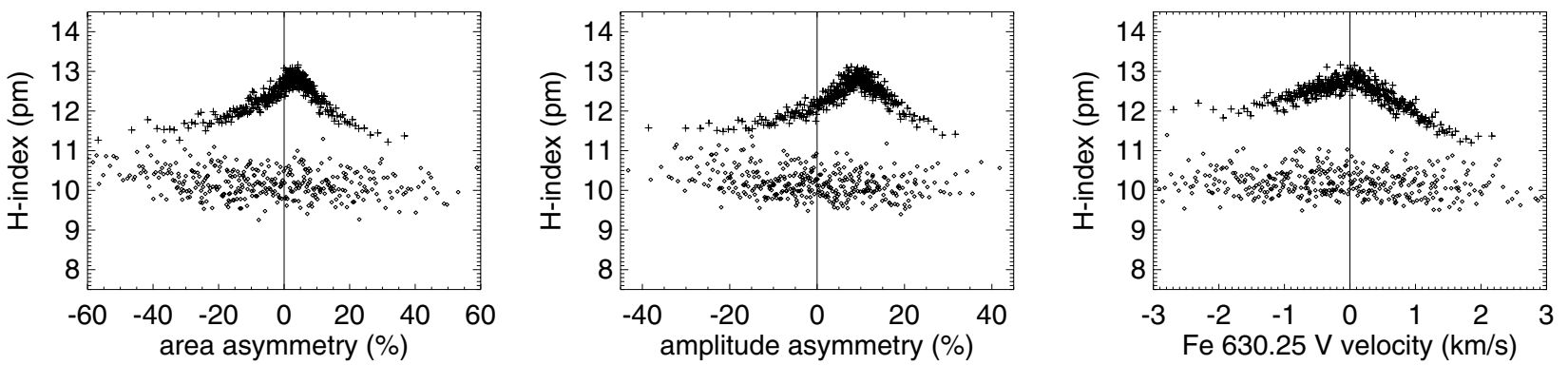

Fig. 9. Correlation between the $H$-index and amplitude/area asymmetry and $V$ velocity. Plusses and squares show network and inter-network, respectively. The binning method is similar to Fig. 8.

Table 4. Fractional distribution of the total $H$-index, $\eta$, for different magnetic flux thresholds.

\begin{tabular}{ccc}
\hline \hline$\Phi_{0}\left(\mathrm{Mx} \mathrm{cm}^{-2}\right)$ & $\#$ & $\eta(\%)$ \\
\hline 4. & 38905 & 63.7 \\
10. & 26071 & 44.5 \\
50. & 11190 & 20.4 \\
100. & 4933 & 9.2 \\
150. & 1709 & 3.3 \\
200. & 329 & 0.6 \\
\hline
\end{tabular}

profiles with a negative $V$ velocity, while in the right panels profiles with a negative area asymmetry are shown. A positive $V$ velocity may thus correspond to a positive or negative area asymmetry. There is no relation between the amplitude/area asymmetries and the $V$ signal in the inter-network (right panels, Fig. 7).

\section{The magnetically and non-magnetically heated components}

The $H$-index includes the $H_{1}$ (the minima outside the emission peaks), $H_{2}$, and $H_{3}$ spectral regions. So its formation height extends from the higher photosphere to the middle chromosphere.

The Ca II H \& K lines are one of the main sources of the chromospheric radiative loss. We use the $H$-index as a proxy for the emission in the low/mid chromosphere, and assume a linear relation between the $H$-index and the chromospheric radiative loss. To derive the contribution of magnetic fields to the chromospheric emission, we added the $H$-index of all points with magnetic flux above a given flux threshold. The fractional $H$-index, $\eta$, is then defined by normalizing this quantity to the total $H$-index of all points in the field of view ${ }^{5}$ :

$\eta\left(\Phi>\Phi_{0}\right)=\frac{\sum H \quad\left(\Phi>\Phi_{0}\right)}{\sum H \quad \text { (allmaps) }}$

Table 4 lists the fractional $H$-index in different magnetic flux thresholds. Some $20 \%$ of the total $H$-index is provided by strong flux concentrations $\left(\Phi \geq 50 . \mathrm{Mx} \mathrm{cm}^{-2}\right)$ while the remaining $80 \%$ of that is produced by the weak magnetic field and/or field free regions.

The average $H$-index is 12.0 and $9.8 \mathrm{pm}$ in the network and inter-network, respectively. However, it contains some contributions from the photosphere (outside emission peaks) and a cool chromosphere (without temperature rise). Therefore, we decompose the observed calcium profiles in a heated and a non-heated component,

$H_{\mathrm{i}}=H_{\mathrm{co}}+H_{\text {non }}+H_{\text {str }}$

${ }^{5}$ We use all data points in this part and do not use the mask defined in Sect. 3.
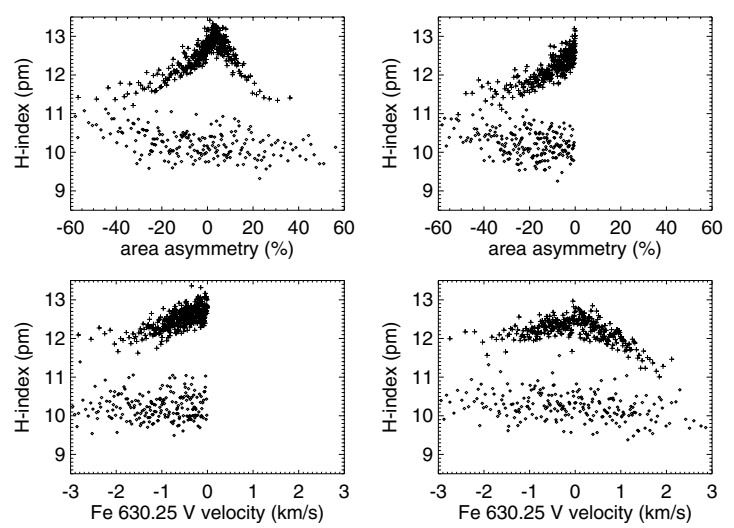

Fig. 10. In the left column diagrams, only the points with a negative $V$ velocity are plotted. In the right panels, only points with a negative area asymmetry are plotted. Pluses and squares show network and internetwork, respectively.

where $H_{\mathrm{i}}$ is the average $H$-index of the inter-network, $H_{\text {co }}$ is the non-heated component, $H_{\text {non }}$ is the non-magnetically heated component, and $H_{\text {str }}$ is the straylight contribution. The nonheated component of the profile originates from the line-wing emission (photosphere) and a cool chromosphere, $H_{\mathrm{co}}$.

We use the Holweger \& Müller (1974) model atmosphere as a proxy for those parts of the solar atmosphere that are not affected by any heating process. This model is very similar to a theoretical radiative equilibrium model, but it was not constructed to strictly satisfy this condition. We prefer this semi-empirical model over a theoretical radiative equilibrium model because it results in slightly better reproduction of the $\mathrm{Ca}$ II $\mathrm{H}$ line wings in a standard disk-center atlas. We note, however, that 3D (M)HD simulations of the solar atmosphere commonly produce regions in the upper photosphere and chromosphere with temperatures significantly lower than in the Holweger-Müller model. These structures, however, are generally so small that their evolution is strongly influenced by their surroundings, so that they cannot serve as a model for those parts of the solar atmosphere that are not influenced by any heating processes. That means that they are not suitable for determining the absolute minimum value of the $H$-index.

We perform a consistent NLTE radiative transfer computation in plane-parallel geometry, including effects of partial frequency redistribution, in order to estimate the non-heated component of the $H$-index. We added an isothermal hydrostatic extension up to a height of $2000 \mathrm{~km}$ to the model in order to be able to compute the core parts of the Ca II H \& K lines. This may seem an arbitrary choice, but considering that the computed intensity is essentially zero at the line core for models with such low temperatures in the upper atmosphere, its influence on the 
$H$-index is very small. This model atmosphere and the obtained profile are similar to the COOLC model of Ayres et al. (1986) and the corresponding profile. We find a value of $H_{\mathrm{co}}=5 \mathrm{pm}$ at disk center. At a heliocentric angle of $53^{\circ}$, the appropriate value is $H_{\text {co }}=5.9 \mathrm{pm}$.

We find an upper limit of $\sim 12 \%$ for the straylight contamination of the observed profiles (cf. Appendix B). Therefore, we decompose the average $H$-index in the inter-network $\left(H_{\mathrm{i}}=9.8 \mathrm{pm}\right)$ to obtain the non-magnetically heated component, $H_{\text {non }}$ :

$H_{\text {non }}=H_{\mathrm{i}}-H_{\mathrm{co}}-H_{\mathrm{str}}$

$H_{\text {non }}=9.8( \pm 1.0)-5.9\left(\begin{array}{l}+0.6 \\ -0.0\end{array}\right)-1.1( \pm 0.1)=2.8\left(\begin{array}{c}+1.1 \\ -1.0\end{array}\right)$.

We find an average non-magnetic heating of $H_{\text {non }} \sim 2.8 \pm 1.1 \mathrm{pm}$. To decompose the average $H$-index of the network, we also consider the magnetically heated component, $H_{\mathrm{mag}}$, and use the same method:

$H_{\text {mag }}=H_{\mathrm{n}}-H_{\mathrm{co}}-H_{\text {non }}-H_{\text {str }}$

where the magnetically heated contribution is the first term in Eq. (1). Assuming a similar non-magnetically heated contribution, $H_{\text {non }}$, for the network as in the inter-network (which is supported by observations, e.g., Schrijver 1987, 1995), we find an average magnetically heated component of about $H_{\mathrm{mag}} \sim$ $1.9 \pm 1.4 \mathrm{pm}$, which is consistent with the difference between the mean $H$-index in the network and inter-network. Thus, the non-magnetically heated component has a larger contribution in the observed $H$-index than magnetically heated one, both in the network and inter-network. Our error estimates in Appendix B show that the uncertainties of the measurements do not affect this result.

\section{Discussion}

\subsection{Thermal coupling}

All intensity parameters, from the calcium core to the Fe I $630 \mathrm{~nm}$ continuum, show different distributions in the network and inter-network: the network patches are brighter not only at all observed wavelengths of the calcium profile, but also in the $630 \mathrm{~nm}$ continuum (Figs. 7 and 4). As noted by Cram \& Damé (1983), this implies that the temperature fluctuations are spatially coherent between the photosphere and the lower chromosphere. This coherent intensity increase is presumably due to the fact that the optical depth scale is shifted downwards in the evacuated magnetic part of the atmosphere.

The correlation between the inner wing intensity and $H$-index is strong, both in the network and inter-network (Table 2). However, the correlation between the outer wing intensity and the $H$-index is significant only in the network (Fig. 6, lower left panel). This indicates that the height range of the thermal coupling between the photosphere and low/mid chromosphere increases in presence of a magnetic field. There are also suggestions that this coupling extends to the upper chromosphere (Rauscher \& Marcy 2006).

\subsection{H-index vs. magnetic flux}

The amount of energy deposited in the network depends on the magnetic flux density. To reproduce the dependence of calcium core emission on the magnetic flux with a power law, we find that the exponent derived strongly depends on the inclusion (or exclusion) of the weakest fluxes. We obtain a power exponent, $b$, of about 0.3, which is smaller than the value given by Schrijver et al. (1989) and Harvey \& White (1999). We ascribe the difference to the higher quality of our data set: better spatial resolution, a lower detection limit for magnetic signals, an accurate estimate of the magnetic flux due to the inversion of vector polarimetric data instead of a magnetogram, and unlike these authors, we do not consider an assumption for the background component prior to the fit. Instead, we keep it as a free parameter. In this way, we find a background component close to the mean $H$-index of the inter-network for the weakest flux concentrations.

The rate of increase of the chromospheric emission vs. magnetic flux reduces for strong magnetic flux densities. This implies that for large flux concentrations, either the filling factor or the magnetic field strength saturates. There are indications that it is the filling factor: the available space is completely filled by expanding flux tubes (Hammer 1987; Saar 1996; Schrijver et al. 1996). In contrast, a lower limit of chromospheric emission also exists. In the inter-network, no relation between emission and the photospheric fields is found (Figs. 8 and 9). The average value of the $H$-index in the inter-network of around 10 pm corresponds to the offset, $c$, Eq. (1). This reflects a constant contribution to the $H$-index which is present even without photospheric magnetic flux, in agreement with Schrijver $(1987,1995)$ who argued that the basal flux does not depend on the magnetic activity. The basal flux contains two components: the non-heated (cf. Sect. 6) and the non-magnetically heated contributions. The non-heated contribution depends on the temperature stratification, so that we speculate that the non-magnetically heated component has an inverse dependence on the temperature stratification.

Figure 8 (upper panel) shows that there is a variable lower limit for the $H$-index versus the magnetic flux density. In contrast, the upper limit is less clearly defined, and would be in agreement with a constant maximum value independent of the amount of magnetic flux. There are also similar behaviors for the upper and lower limits in the scatter plots of the $H_{3}, H_{2 \mathrm{v}}$, and $H_{2 r}$ versus the magnetic flux density. This is similar to upper and lower limits of the basal flux of stars vs. the color $(B-V)$ where the lower boundary changes but the upper one is almost constant (Fawzy et al. 2002b, their Fig. 2). The situation for the inter-network is different: both the upper and lower limits are independent of the magnetic flux density.

\subsection{Decomposing the Ca II H profile}

We interpret the solar $\mathrm{Ca}$ II $\mathrm{H}$ line profile as the superposition of a cool chromospheric profile, the heated component and straylight components. Oranje (1983) first applied this method to reproduce different observed Ca II K profiles from two "basic" profiles. Later, Solanki et al. (1991) used the same idea to decompose the observed profiles by a combination of two theoretically calculated profiles. Our one-dimensional NLTE radiative transfer calculations reveal that the $H$-index of a cool chromosphere is about $5.9 \mathrm{pm}$. Since the chromospheric emission of the Sun as a star is slightly above the minimum among the Sunlike stars (Schrijver \& Zwaan 2000), the average quiet Sun profile contains a heated component. Subtraction of the cool chromospheric component from the measured $H$-index (along with straylight considerations) leads to an estimation of the pure nonmagnetically and magnetically heated components in the measured $H$-index. The non-magnetically heated component is almost $50 \%$ larger than the magnetically heated component. The presence of a significant non-heated contribution in the $H$-index indicates that not all of the chromospheric emission emerges 
from a hot chromosphere, in contrast to findings from, e.g., Kalkofen et al. (1999). The minimum heated component in a $\mathrm{Ca}$ profile provides evidence how cool the chromosphere may be (e.g., Wedemeyer-Böhm et al. 2005). Moreover, it favors theories in which the non-magnetic heating plays the dominant role in the chromospheric heating (Fawzy et al. 2002a).

The fact that the non-magnetic chromospheric heating contributes significantly to the chromospheric energy balance is also found in the relative contributions of mainly field-free internetwork and network areas to the total emission in the field of view. Magnetic flux densities above $50 \mathrm{Mx} \mathrm{cm}^{-2}$ add only $20 \%$ to the total emission, which is also seen in the ratio of the mean $H$-index in the network and inter-network, $12.0 / 9.8 \approx 1.2$ (Table 4).

\subsection{Uncertainties}

The main uncertainty in the non-magnetically and magnetically heated components is the presence of some heating in our cool chromosphere profile. The Holweger \& Müller (1974) model atmosphere is close to an atmosphere in radiative equilibrium without non-magnetic or magnetic heating, so no heated component (cf. Sect. 6) is expected to be found on its Ca profile. Since there is no general agreement how cool the chromosphere may be (e.g., Kalkofen et al. 1999; Ayres 2002), the best answer would be to use the observed calcium profile with the lowest $H$-index. However, Solanki et al. (1991) concluded that it is not possible to observe a low activity profile as presented by the COOLC model of Ayres et al. (1986), which is similar to the one employed by us on base of the Holweger \& Müller (1974) model atmosphere. Thus, our cool profile can well serve as lower limit of the non-heated contribution to the $H$-index. Note that a larger value for $H_{\text {co }}$ would increase the contribution of the magnetically heated component, leading to a larger fractional contribution for the magnetically heated to the total heated component.

There may be some mixed polarity fields below our polarimetric detection limit which may influence the ratio of the magnetically heated to the non-magnetically heated component.

\subsection{H-index vs. V asymmetries}

From the relations of the chromospheric emission to quantities of the photospheric magnetic field in the network, we find that the chromospheric emission peaks at small positive values of the Stokes $-V$ asymmetries and at zero Stokes $-V$ velocity (Fig. 5). It reflects the histograms of the respective photospheric field quantities which show similar distributions even at disk center (e.g., Sigwarth et al. 1999). However, in combination with the dependence of the emission on the flux density it is inferred that the stronger flux concentrations mainly show small material flows along with non-zero asymmetries.

The magnetically heated component is related to Stokes- $V$ profiles with non-zero area and amplitude asymmetries (Figs. 5 and 9). A possible explanation for this finding would be the absorption of upward propagating acoustic waves, generated by the turbulent convection, by the inclined fields of expanding flux tubes. This would imply that the energy is deposited at the outer boundary or in the canopy of flux concentrations rather than in the central, more vertical, part. It is mainly because the non-magnetic cutoff frequency is lowered at the boundary of the flux tubes, where the field lines are inclined. This was first predicted by Suematsu (1990) and recently achieved some observational support (Hansteen et al. 2006; Jefferies et al. 2006).
Figure 9 indicates that the maximum observed $H$-index has nonzero $V$ asymmetry. These asymmetric $V$ profiles are consistent with the case when the line of sight passes through the canopy of a magnetic element or through a flux tube axis (positive and negative asymmetries respectively, Steiner 1999). Therefore our finding supports Suematsu (1990).

\subsection{Inter-network field strength distribution}

As a byproduct of our study on the relation between photospheric fields and chromospheric emission, we obtained distributions of field strength for network and inter-network regions. For the inter-network fields, for the first time an inversion of visible spectral lines in the weak field limit led to the same distribution as results from the more sensitive infrared lines (Collados 2001). This is discussed in Appendix A. Thus, we find that the inter-network magnetic field is dominated by field strengths of the weak field regime which ranges up to some $600 \mathrm{G}$. This rules out the argument that the inter-network is dominated by a magnetic field with a strength of more than a kilo-Gauss (e.g., Sánchez Almeida et al. 2003a).

\section{Conclusions}

There is no correlation between the $H$-index and magnetic field parameters in the inter-network. Therefore, we conclude that the magnetic field has a negligible role in the chromospheric heating in the inter-network. On the other hand, the $H$-index is a power law function of the magnetic flux density in the network with a power index of 0.3 . The average $H$-index observed in the network and inter-network are $\sim 10$ and $12 \mathrm{pm}$, respectively. We find a non-magnetic component in the network (based on Eq. (1)) of about $10 \mathrm{pm}$ which shows the consistency of our analysis: the non-magnetic part of the network $H$-index is equal to the $H$-index of the inter-network.

A NLTE radiative transfer calculation, using the HolwegerMüller model atmosphere, indicates that the non-heated component of the $H$-index, emerging from a cool chromosphere, is about $5.9 \mathrm{pm}$. Comparison of this non-heated component and the average $H$-index in the inter-network has two implications: a) some of the observed chromospheric emission does not originate from a hot chromosphere, and b) the nonmagnetically heated component is about $50 \%$ larger than the magnetically heated component. From this, we conclude that the non-magnetically heated component has a larger contribution in the chromospheric radiative loss than the magnetically heated component, both in the network and inter-network.

In our statistical ensemble, spatial positions with strong magnetic field $\left(\Phi \geq 50 \mathrm{Mx} \mathrm{cm}^{-2}\right)$ contribute about $20 \%$ of the total $H$-index. Correlations and histograms of the different intensity bands in the $\mathrm{Ca}$ II H spectrum indicate that above a magnetic threshold, photosphere and low/mid chromosphere are thermally coupled. Moreover, our findings are consistent with the idea that the energy transfer in a flux tube has a skin effect: the energy transfer is more efficient in the flux tube boundary (canopy) than at its center (axis).

For the first time, we find a magnetic field distribution in the inter-network using visible lines which is similar to results inferred from infrared lines with larger Zeeman splitting. The distribution function increases with decreasing field strength. The peak at $200 \mathrm{G}$ is due to the detection limit of the polarization signal. The average and distribution peak of the absolute flux density (of magnetic profiles) in the inter-network are $\sim 11$ and 


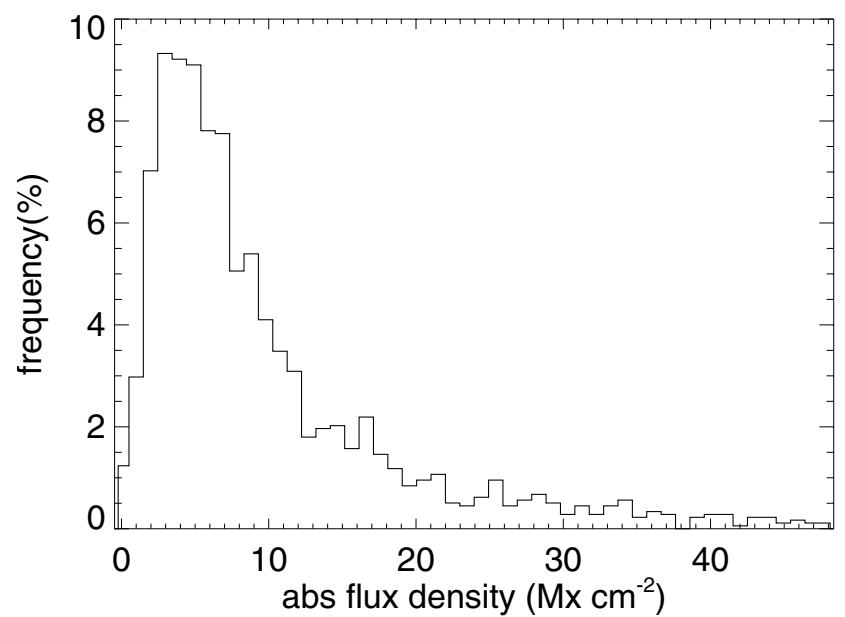

Fig. A.1. Close up view of the flux density distribution for the internetwork. At a value of $\sim 4.0 \mathrm{Mx} \mathrm{cm}^{-2}$, the histogram shows a peak.

$4 \mathrm{Mx} \mathrm{cm}^{-2}$, respectively. We conclude that the combination of high spatial resolution and polarimetric accuracy is sufficient to reconcile the different results on field strength found from infrared and visible lines.

Acknowledgements. The principal investigator (PI) of the ITP observing campaign was P. Sütterlin, Utrecht, The Netherlands. We wish to thank Reiner Hammer, Oskar Steiner, Thomas Kentischer, Wolfgang Rammacher, and Hector Socas-Navarro for useful discussions.The POLIS instrument has been a joint development of the High Altitude Observatory (Boulder, USA) and the Kiepenheuer-Institut. Part of this work was supported by the Deutsche Forschungsgemeinschaft (SCHM 1168/8-1).

\section{Appendix A: The magnetic field distribution}

Traditional magnetic field strength distributions for the quiet Sun (inter-network) based on visible lines have a broad peak centered around $1 \mathrm{kG}$ (Sánchez Almeida et al. 2003a; Domínguez Cerdeña et al. 2006). On the other hand, observations in the infrared lines show a clear peak at lower field strengths and an almost exponentially decreasing probability for higher fields strengths (Collados 2001; Bellot Rubio \& Collados 2003; Khomenko et al. 2003). This discrepancy between the retrieved parameters from the visible and infrared lines has not been solved so far (Steiner 2003; Martínez González et al. 2006).

We achieved a magnetic field distribution for the internetwork using the Fe I $630 \mathrm{~nm}$ pair that is similar to the infrared studies (Fig. 5b). We elaborate on the effects of spatial resolution and signal-to-noise ratio on this finding. We used the Kiepenheuer Adaptive Optics System (KAOS) to improve spatial resolution and image stability (von der Lühe et al. 2003). It provides stable sharp images in a certain field of view, and therefore allows us to increase the exposure time to have better signal-to-noise ratio.

Figure A.1 is a close-up view of the inter-network magnetic flux distribution. The lowest flux detected is $\sim 0.05 \mathrm{Mx} \mathrm{cm}^{-2}$. The distribution of the flux decreases sharply for fluxes below $\Phi \leq 4 \mathrm{Mx} \mathrm{cm}^{-2}$, which is due to the detection limit of the polarization signal. The small fluxes still detected give us, however, confidence that the magnetic field strength distribution of Fig. 5b is reliable as a consequence of the combination of high spatial resolution and good signal-to-noise ratio.

To investigate the influence of noise on the inversion results, we compare inversion results of the original datasets with and without adding noise. In order to create "noisy" data sets, the
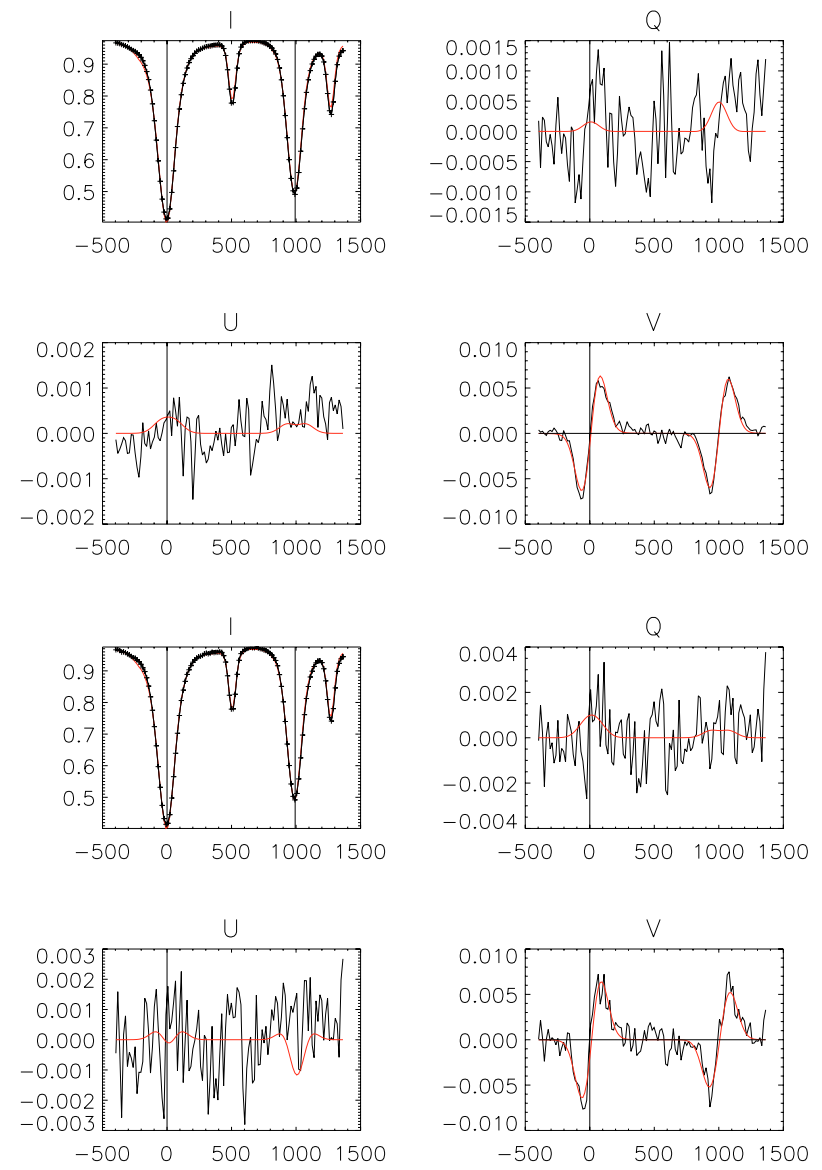

Fig. A.2. The upper four plots show an example of the original Stokes profiles and the inversion fits. The lower four figures show the same profile with a rms noise value two times larger and corresponding fits. The magnetic field strength, magnetic filling factor, and macro turbulence velocity for the original and noisy profiles are $(842,1543) \mathrm{G}$, $(7.6,5.3) \%$, and $(2.95,2.77) \mathrm{km} \mathrm{s}^{-1}$ respectively. The $x$-axis shows the spectral coordinate in $\mathrm{m} \AA$.

rms value of the noise in the original data was increased to $\sigma=15.0 \times 10^{-4} I_{\mathrm{c}}$, two times larger than the original value. The spatial sampling was identical to the original data. We emphasize that inversion was done using the same assumptions and initial model atmosphere. Figure A.2 compares two sets of full Stokes profiles (black) and the inversion fit (red). The upper four panels are the original profiles and the lower four panels are the same profiles with noise. The fits and retrieved model atmospheres differ significantly. Especially important is the fact that the noisy inversion leads to a magnetic field of $1.5 \mathrm{kG}$.

To compare the field strength obtained from the original and noisy datasets, their histograms are shown in Fig. A.3. The distribution of the original field strength peaks around $\sim 200 \mathrm{G}$ (black histogram), while for the noisy data, it has a clear shift toward higher values with a peak at $0.8 \mathrm{kG}$ (red histogram). This emphasizes the role of the noise in the existing discrepancy between visible and infrared measurements (Bellot Rubio \& Collados 2003).

In brief, we find that higher spatial resolution of these observations along with low noise data is an important step toward resolving disagreements between the visible and infrared polarimetric measurements. The visible Fe I $630 \mathrm{~nm}$ pair shows higher Stokes amplitudes for small magnetic fields than the infrared lines of Fe I $1.56 \mu \mathrm{m}$. Hence, with an equal amount of noise in observational data, the visible lines provide a higher 


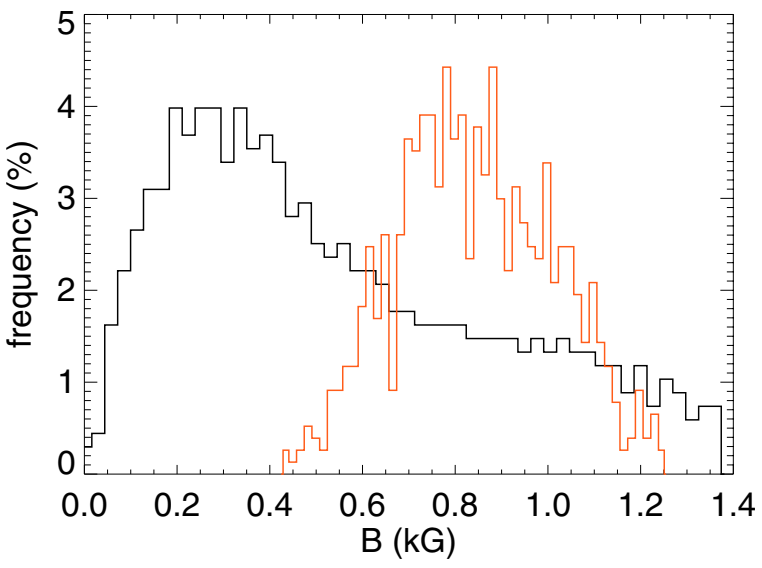

Fig. A.3. Histograms of the inter-network field strengths of one of the maps based on the inversion of original data (black) and noisy data (red).

signal-to-noise ratio than infrared lines. Therefore, in contrast to Martínez González et al. (2006), we find the visible Fe I 630 nm pair to be a proper tool to investigate the inter-network magnetic field. We ascribe the present disagreements to the low spatial resolution of previous observations and different signal-to-noise ratios.

\section{Appendix B: Calibration uncertainties in POLIS Ca channel}

The POlarimetric LIttrow Spectrograph (POLIS) was designed to facilitate co-temporal and co-spatial measurements of the vector magnetic field in the photosphere and the $\mathrm{Ca}$ II H intensity profile (Schmidt et al. 2003; Beck et al. 2005a). We try to elaborate on the uncertainties in the intensity calibration, because it is critical for the estimates of the various contributions (straylight, non-magnetic heating, etc.) to the observed calcium profiles. Observations are discussed in Sect. B.1. We study the linearity of the CCD camera for different light levels in Sect. B.2. Then, we investigate instrumental and solar straylight contributions in Sect. B.3. In Sect. B.4, we perform standard error propagation to quantify uncertainties in our final calibrated spectra.

\section{B.1. Observations}

In order to study linearity of the POLIS CCD camera of the calcium channel at different light levels, we observed a set of calcium spectra and flat field data with different accumulations and slit widths at disk center. A sunspot was also observed with a similar setup and an exposure time of $0.82 \mathrm{~s}$ and six accumulations. Besides this, we recorded dark current data using either the $\mathrm{AO}$ or POLIS field stop. We use these two data sets to estimate the solar and instrumental straylight, respectively.

All spectra and profiles in this Appendix are dark subtracted raw data. No other calibration process like flat fielding was applied. In order to show intrinsic noise in the data, we did not spatially average darks or spectra.

\section{B.2. Linearity}

In order to check the linearity of the calcium camera from profiles by, e.g., comparing the ratio of the core to wing for different exposures, we recorded spectra with different slit widths and identical modulation speed (Mullikin et al. 1994). Figure B.1

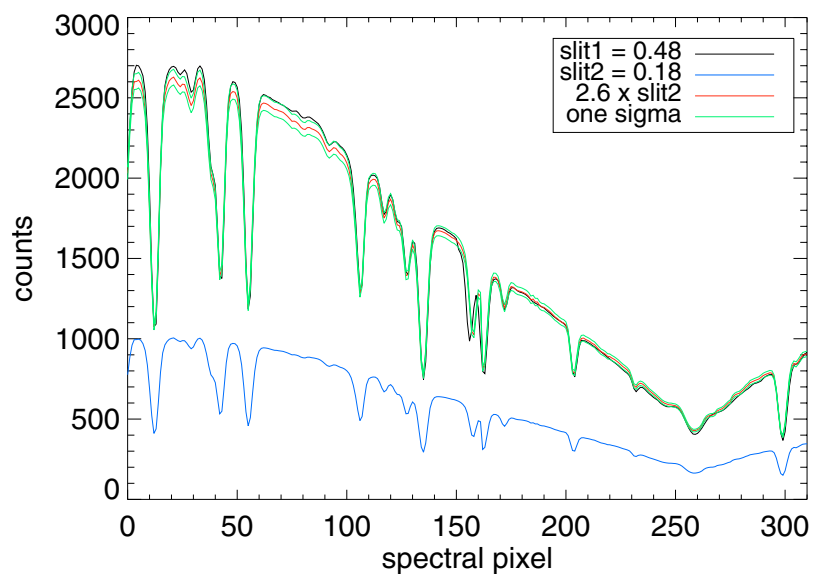

Fig. B.1. Comparison between two different slit widths and the same modulation speed. The black and blue profiles correspond to slit widths of 0.48 and 0.18 arcsec, respectively. The red profile is the blue profile multiplied by a constant factor of 2.6 for all pixels. The green profiles show the $1-\sigma$ rms value $(\sim 1 \%)$ from the mean curve (red).

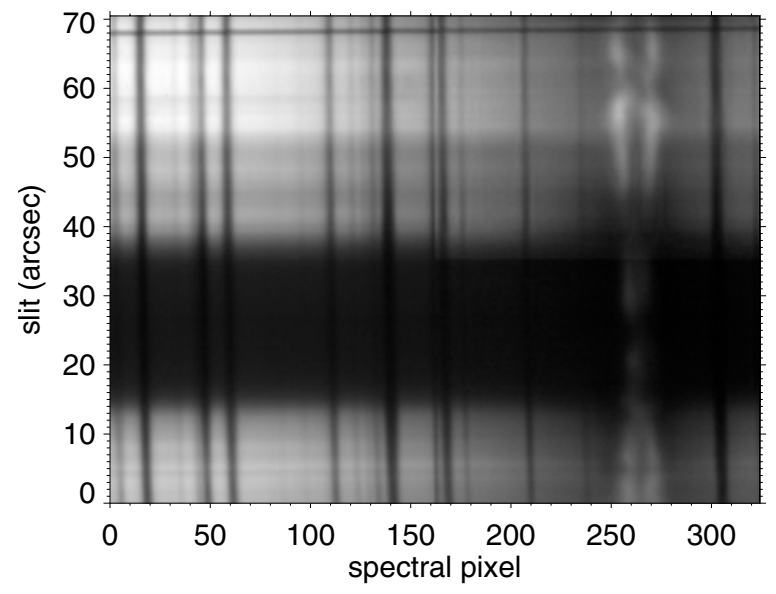

Fig. B.2. Dark subtracted spectrum of the umbra in a sunspot.

shows a comparison between the two flat field profiles taken on disk center with identical illumination, but different slit widths. The black and blue profiles correspond to a slit width of 0.48 and 0.18 arcsec, respectively. If one multiplies the blue curve with the ratio of the slit widths of 2.67, the resulting curve agrees well with the profile of the broad slit for all wavelengths including the line core. We calculated the ratio of the two profiles with different slit widths as function of wavelength. The rms variation of the ratio was $1 \%$. This can be used as an upper limit for non-linearity effects of the CCD camera.

\section{B.3. Straylight}

\section{B.3.1. False light}

We compare two sets of dark current images of the POLIS Fe channel: one with a field stop right after the focal plane, and another one with the field stop in front of the camera. In the second case, all light sources inside the observing room are fully blocked. The difference between the two measurements is about $0.9 \pm 0.5 \%$. This gives an upper limit for the instrumental straylight in the POLIS Ca channel as well, since most of the sources in the observing room will not emit in blue as efficient as red. 

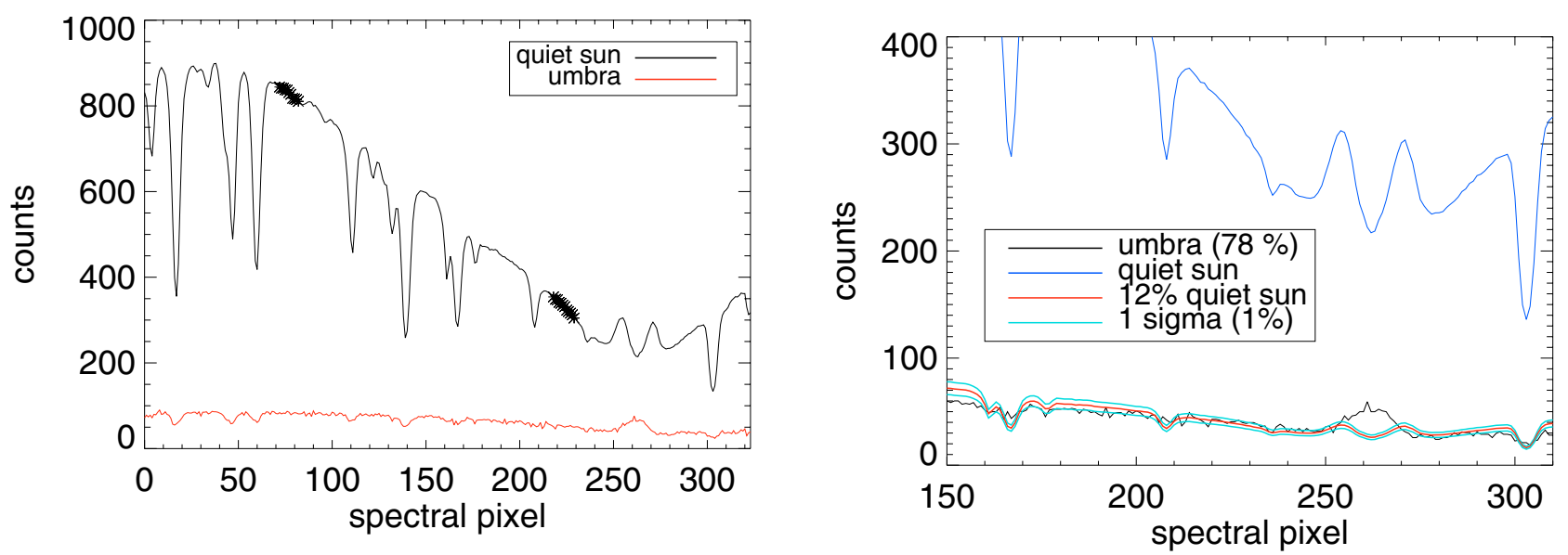

Fig. B.3. Left: a single $\mathrm{Ca}$ II $\mathrm{H}$ spectrum of the umbra and quiet sun profiles in a sunspot. Asterisks show spectral bands that were used to estimate the true signal (see Sect. B.3.2). Right: close up view of the quiet sun and umbral profiles near the calcium line core. The true signal (about $5 \%$ in the wing close to the Ca core according to Wallace et al. (2000) was subtracted from the umbral profile. The red curve shows $12 \%$ of the quiet sun profile. The two cyan curves show an uncertainty interval of one sigma $(1 \%)$.

\section{B.3.2. Atmospheric and telescopic straylight}

The calcium profile in the sunspot umbra is significantly weaker than a quiet sun profile. However, there is a well known single emission peak in the calcium core (Linsky \& Avrett 1970). We thus compare an inner wing band close to the calcium core, but excluding the core itself (asterisks in the left panel of Fig. B.3), in an umbral profile and a normal profile from outside the spot to derive an upper limit for the solar straylight contribution to observed profiles.

Figure B.2 shows a slit spectrum of the umbra. To keep the sample profiles as uniform as possible, we chose two identical positions along the slit (at about $30 \mathrm{arcsec}$, Fig. B.2). The spectrum of the quiet sun and the minimum spectrum of the umbra are shown in Fig. B.3 (left panel).

Wallace et al. (2000) presented a Fourier transform spectral atlas of the umbra. In this atlas, the wing close to the umbral core has an intensity of $5 \%$ in units of the umbral continuum, while the wing close to the Fe I, Ti I, and Ni I lines at about $396.5 \mathrm{~nm}$ has an intensity of $38 \%$. In our umbral profile, this continuum has an intensity of about 80 counts (asterisks, Fig. B.3, left panel), whereas close to the core the profile has about 45 counts. Assuming no straylight in the atlas profile, only 11 counts ( $\sim 22 \%$ of the observed valued) should be measured in the core. Thus, $78 \%$ of the observed signal have to be due to straylight. To reproduce $78 \%$ of the umbral profile, a straylight contribution of around $12 \%$ is needed. If we assume that there is no real calcium signal in the umbra (which is wrong) and take the observed calcium profile of the umbra as pure scattered light, the ratio changes from $12 \%$ to $15 \%$. Therefore, uncertainties about the scattered light in the atlas profile have minor importance.

A close-up view of the spectral region close to the calcium core is shown in Fig. B.3 (right panel). For the reason mentioned above, in the right panel of Fig. B.3, the umbral profile was labeled umbra (78\%). The red curve shows $12 \%$ of the quiet sun profile. The two cyan curves also show an uncertainty interval of one sigma (1\%). Figure B.4 shows the ratio of the umbra to the quiet sun profile. If we calculate mean and rms of the whole spectrum, it gives a value of $10 \pm 3 \%$ while considering only on the wing band close to the calcium core, we obtain $12 \pm 1 \%$. We take this value as an upper limit for the total straylight in the POLIS calcium channel. In Sect. B.3.1, we concluded that the instrumental straylight is about $1 \%$. Hence, most of the obtained

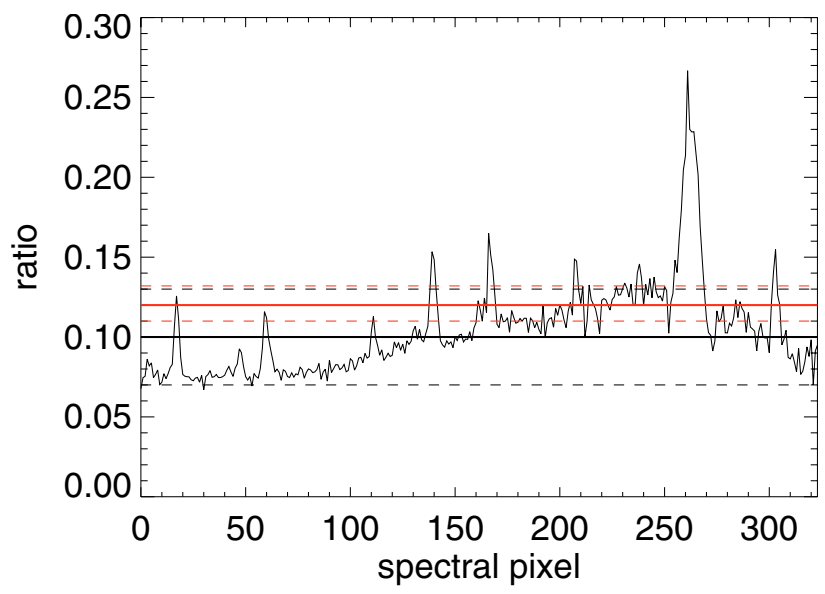

Fig. B.4. Ratio of the umbral profile to a quiet sun profile. If one considers all the spectrum, the ratio will be $10 \pm 3 \%$ while considering only parts of the wing close to the $\mathrm{CaIIH}$ core, the ratio will be $12 \pm 1 \%$. Note to the mismatch between the quiet sun and umbral profiles at spectral line positions due to different broadenings.

straylight originates from the scattered solar light in the telescope and the earth atmosphere.

\section{B.4. Noise}

There are three noise sources in a CCD camera (Mullikin et al. 1994):

1. readout noise because of the pre-amplifier;

2. dark current noise which is influenced by the chip temperature;

3. photon noise due to the quantum nature of light which obeys Poisson statistics.

The readout error of the camera was estimated by a set of 50 consecutive dark frames with similar exposure and accumulations. Here, we compare the number of counts on each pixel in different images. We attribute the standard deviation of the count value mainly to the readout error, and hence, obtain a readout error for each pixel separately. The distribution of the readout errors is shown in Fig. B.5. The readout noise is about $\sigma_{\text {readout }} \sim 2$ counts. 


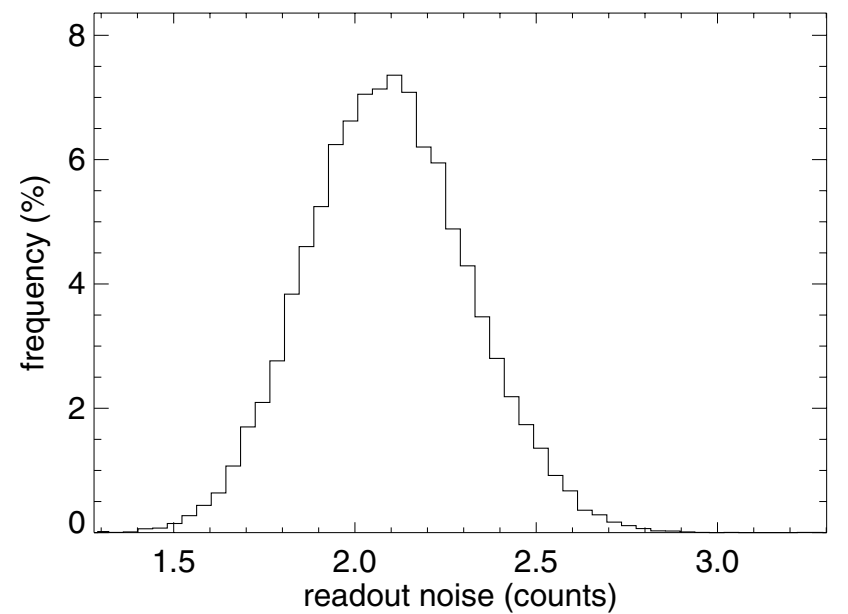

Fig. B.5. Distribution of the readout error in a set of 50 dark currents with 8 accumulations.

To investigate the thermal noise in the dark current, one has to remove small systematic offsets between CCD columns (probably due to thermal fluctuations of the AD system) before considering statistical measures, e.g., rms, of a dark profile (a CCD row). As it is shown in Fig. B.6, for a dark frame with 6 accumulations, the resulting rms (after removing systematics) is far less than the standard deviation of the whole map including a large-scale variation. It provides an estimate for the thermal noise in the camera. So the rms of the dark frame is about $\sigma_{\text {thermal }} \sim 3$ counts.

The total noise in the recorded data, $\sigma_{\text {noise }}$, consists of three components:

$\sigma_{\text {noise }}=\sqrt{\sigma_{\text {photon }}^{2}+\sigma_{\text {thermal }}^{2}+\sigma_{\text {readout }}^{2}}$

where the photon noise originates from the Poisson fluctuations of the number of photons counted. Assuming that $n_{\text {photon }}$ is the number of detected photons, the corresponding $\mathrm{rms}, \sigma_{\text {photon }}^{2}=$ $n_{\text {photon}}$. The calcium core is more influenced by the noise than the other points in the spectrum. Figure B.7 shows a dark subtracted calcium spectrum with 6 accumulations and a narrow slit $(0.18 \mathrm{arcsec})$. The minimum number of counts in the core is 117 , so $\sigma_{\text {photon }} \sim 11$. Therefore, the total noise in the core (Eq. (B.1)) will be $\sigma_{\text {noise }} \sim 11$. So the worst signal-to-noise ratio at the calcium core position will be better than 10 . Note that the data described in the paper were obtained with a wide slit $(0.48$ arcsec $)$ while the spectrum shown in Fig. B.7 was recorded with a narrow slit $(0.18 \operatorname{arcsec})$, so we have better signal-to-noise ratio in the data.

\section{B.5. Error propagation}

The relative intensity of the core to wing is defined as:

$x=\frac{I_{\text {core }}}{I_{\text {wing }}}$.

To estimate their rms errors, we find the signal-to-noise ratio for the core and wing intensities in some wing bands. Since it is not possible to use the core intensity to obtain the noise due to intrinsic changes, we use the average signal-to-noise of the left and right wings close to the calcium core as the signal-to-noise of the core. So the real signal-to-noise of the core is probably worse than that. The $\mathrm{S} / \mathrm{N}$ ratio of the core and wing vary from 35
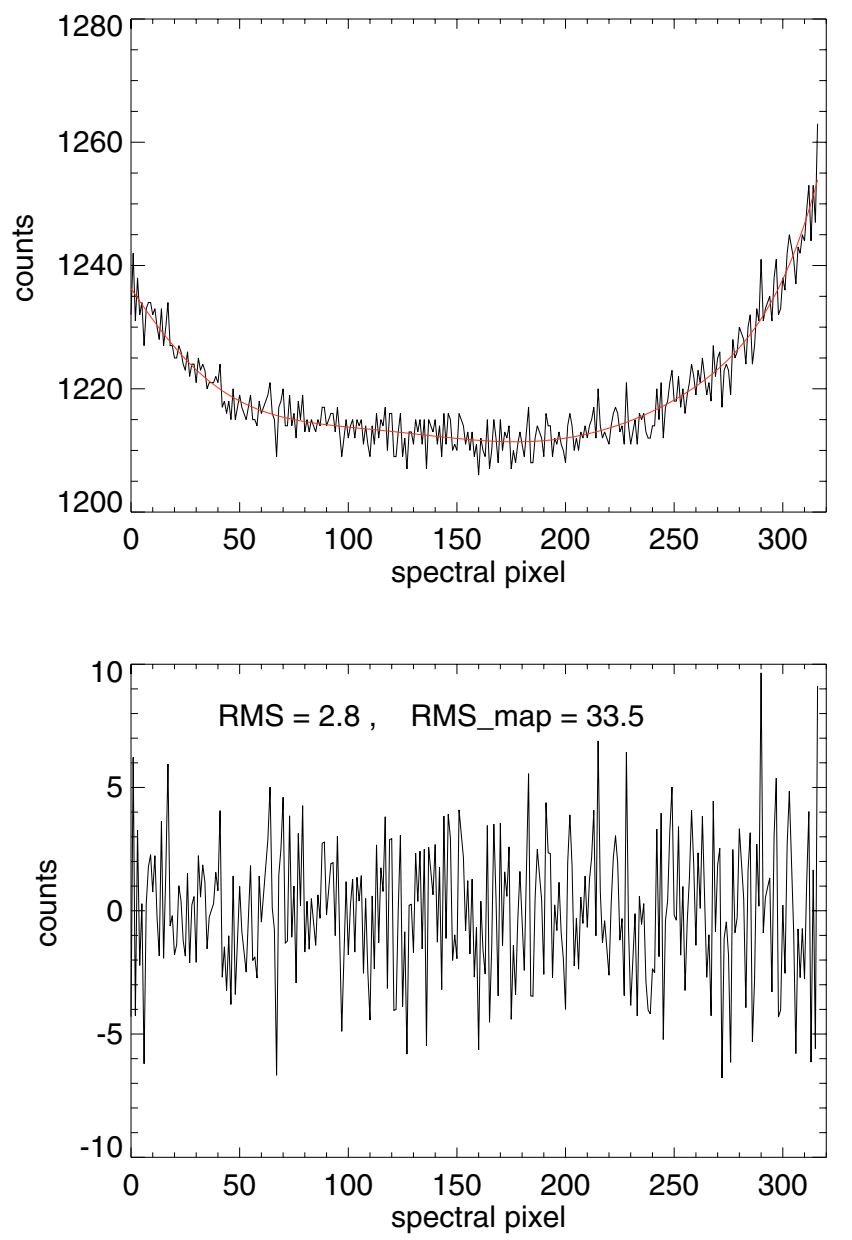

Fig. B.6. Upper panel: a row in a dark frame with 6 accumulation. The red curve is a polynomial fit to subtract systematic shift from the random noise. Lower panel: the residual signal after subtraction of the polynomial, which is the real noise. Note to the large difference between the rms of the map (excluding its borders) and the rms of the residuals.

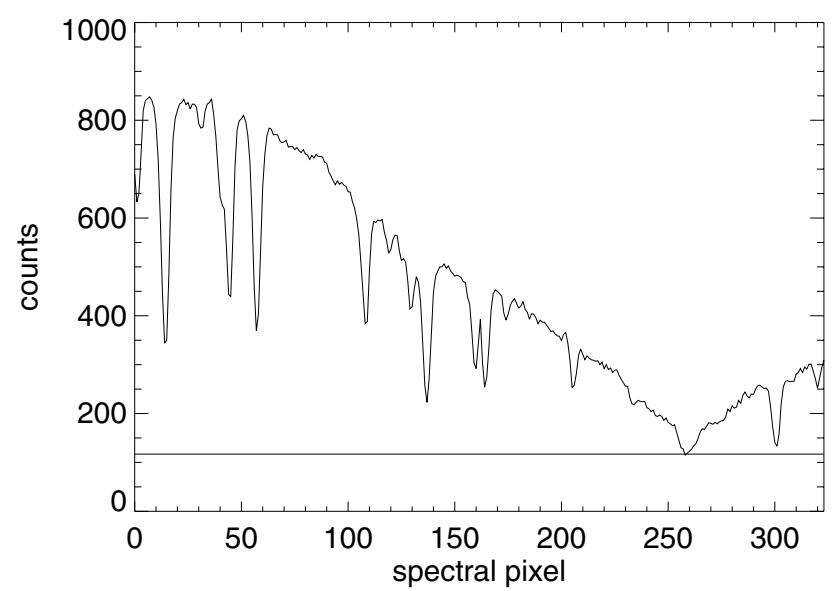

Fig. B.7. A sample calcium profile. We use the calcium core minimum counts to estimate the photon noise. In this case, the signal-to-noise ratio at the calcium core is more than 10 .

and 112 for two accumulations to 174 and 216 for twenty accumulations. We use the worse $\mathrm{S} / \mathrm{N}$ ratio corresponding to an 
exposure time of $1.64 \mathrm{~s}$ and obtain the following values for the rms errors of the core and wing intensities:

$$
\begin{aligned}
\frac{\sigma_{\mathrm{c}}}{I_{\text {core }}} & \sim \frac{1}{34} \sim 3 \% \\
\frac{\sigma_{\mathrm{w}}}{I_{\text {wing }}} & \sim \frac{1}{112} \sim 1 \% .
\end{aligned}
$$

So, we can obtain the $\sigma_{x}$ as follows:

$$
\frac{\sigma_{x}}{x}=\sqrt{\left(\frac{\sigma_{\mathrm{c}}}{I_{\text {core }}}\right)^{2}+\left(\frac{\sigma_{\mathrm{w}}}{I_{\text {wing }}}\right)^{2}} \sim 3 \% \text {. }
$$

Error sources of the final calibrated data include uncertainties from the flat-field parameter, the straylight and the linearity of the detector. The straylight uncertainty was obtained about $1 \%$ in Sect. B.3. Assuming an upper limit for the uncertainty in the flatfield to be $5 \%$ and some $1 \%$ uncertainty in linearity of the core to wing ratio, an upper limit for the uncertainty in the calibrated spectrum will be:

$\frac{\sigma_{r}}{r}<10 \%$.

\section{References}

Ayres, T. R. 2002, ApJ, 575, 1104

Ayres, T. R., Testerman, L., \& Brault, J. W. 1986, ApJ, 304, 542

Beck, C., Schlichenmaier, R., Collados, M., Bellot Rubio, L., \& Kentischer, T. 2005a, A\&A, 443, 1047

Beck, C., Schmidt, W., Kentischer, T., \& Elmore, D. 2005b, A\&A, 437, 1159

Beck, C., Bellot Rubio, L., Schlichenmaier, R., \& Sütterlin, P. 2006, A\&A, submitted

Bellot Rubio, L. R., \& Collados, M. 2003, A\&A, 406, 357

Bellot Rubio, L. R., \& Beck, C. 2005, ApJ, 626, L125

Berger, T. E., \& Title, A. M. 2001, ApJ, 553, 449

Carlsson, M., \& Stein, R. F. 1997, ApJ, 481, 500

Cattaneo, F., Emonet, T., \& Weiss, N. 2003, ApJ, 588, 1183

Collados, M. 2001, in Advanced Solar Polarimetry - Theory, Observation, and Instrumentation, ed. M. Sigwarth, ASP Conf. Ser., 236, 255

Cram, L. E., \& Damé, L. 1983, ApJ, 272, 355

de Wijn, A. G., Rutten, R. J., Haverkamp, E. M. W. P., \& Sütterlin, P. 2005, A\&A, 441, 1183

Domínguez Cerdeña, I., Almeida, J. S., \& Kneer, F. 2006, ApJ, 646, 1421

Faurobert, M., Arnaud, J., Vigneau, J., \& Frisch, H. 2001, A\&A, 378, 627

Fawzy, D., Rammacher, W., Ulmschneider, P., Musielak, Z. E., \& Stępień, K. 2002a, A\&A, 386, 971

Fawzy, D., Ulmschneider, P., Stępień, K., Musielak, Z. E., \& Rammacher, W. 2002b, A\&A, 386, 983

Fossum, A., \& Carlsson, M. 2005, Nature, 435, 919

Hammer, R. 1987, Solar and Stellar Physics, LNP, 292, 77

Hansteen, V. H., De Pontieu, B., Rouppe van der Voort, L., van Noort, M., \& Carlsson, M. 2006, ApJ, 647, L73

Harvey, K. L., \& White, O. R. 1999, ApJ, 515, 812

Holweger, H., \& Müller, E. A. 1974, Sol. Phys., 39, 19

Jefferies, S. M., McIntosh, S. W., Armstrong, J. D., et al. 2006, ApJ, 648, L151
Judge, P. G., \& Peter, H. 1998, Space Sci. Rev., 85, 187

Kalkofen, W., Ulmschneider, P., \& Avrett, E. H. 1999, ApJ, 521, L141

Keller, C. U., Deubner, F.-L., Egger, U., Fleck, B., \& Povel, H. P. 1994, A\&A, 286, 626

Khomenko, E. V., Collados, M., Solanki, S. K., Lagg, A., \& Trujillo Bueno, J. 2003, A\&A, 408, 1115

Linsky, J. L., \& Avrett, E. H. 1970, PASP, 82, 169

Lites, B. W. 2002, ApJ, 573, 431

Lites, B. W., Rutten, R. J., \& Kalkofen, W. 1993, ApJ, 414, 345

Lites, B. W., Rutten, R. J., \& Berger, T. E. 1999, ApJ, 517, 1013

Martínez González, M. J., Collados, M., \& Ruiz Cobo, B. 2006, A\&A, 456, 1159

Mullikin, J. C., van Vliet, L. J., Netten, H., et al. 1994, in Image Acquisition and Scientific Imaging Systems, ed. H. C. Titus, \& A. Waks, Proc. SPIE, 2173, 73

Narain, U., \& Ulmschneider, P. 1996, Space Sci. Rev., 75, 453

Oranje, B. J. 1983, A\&A, 124, 43

Priest, E. R., Heyvaerts, J. F., \& Title, A. M. 2002, ApJ, 576, 533

Rauscher, E., \& Marcy, G. W. 2006, PASP, 118, 617

Rezaei, R., Schlichenmaier, R., Beck, C. A. R., \& Bellot Rubio, L. R. 2006, A\&A, 454, 975

Rezaei, R., Schlichenmaier, R., Beck, C. A. R., \& Schmidt, W. 2007, in Modern Solar Facilities-Advanced Solar Science, ed. F. Kneer, K. G. Puschmann, \& A. D. Wittmann, in press

Ruiz Cobo, B., \& del Toro Iniesta, J. C. 1992, ApJ, 398, 375

Rutten, R. J. 1999, in Third Advances in Solar Physics Euroconference:

Magnetic Fields and Oscillations, ed. B. Schmieder, A. Hofmann, \&

J. Staude, ASP Conf. Ser., 184, 181

Rutten, R. J., \& Uitenbroek, H. 1991, Sol. Phys., 134, 15

Saar, S. H. 1996, in Stellar Surface Structure, ed. K. G. Strassmeier, \& J. L. Linsky, IAU Symp., 176, 237

Sánchez Almeida, J., Domínguez Cerdeña, I., \& Kneer, F. 2003a, ApJ, 597, L177

Sánchez Almeida, J., Emonet, T., \& Cattaneo, F. 2003b, ApJ, 585, 536

Schmidt, W., Beck, C., Kentischer, T., Elmore, D., \& Lites, B. 2003, Astron.

Nachr., 324, 300

Schrijver, C. J. 1987, A\&A, 172, 111

Schrijver, C. 1995, A\&A Rev., 6, 181

Schrijver, C. J., \& Zwaan, C. 2000, Solar and Stellar Magnetic Activity (Cambridge University Press)

Schrijver, C. J., Cote, J., Zwaan, C. \& Saar, S. H. 1989, ApJ, 337, 964

Schrijver, C. J., Shine, R. A., Hagenaar, H. J., et al. 1996, ApJ, 468, 921

Sigwarth, M. 2001, ApJ, 563, 1031

Sigwarth, M., Balasubramaniam, K. S., Knölker, M., \& Schmidt, W. 1999, A\&A, 349,941

Skumanich, A., Smythe, C., \& Frazier, E. N. 1975, ApJ, 200, 747

Socas-Navarro, H. 2005, ApJ, 633, L57

Solanki, S. K., \& Steiner, O. 1990, A\&A, 234, 519

Solanki, S. K., Steiner, O., \& Uitenbroeck, H. 1991, A\&A, 250, 220

Steiner, O. 1999, in Third Advances in Solar Physics Euroconference: Magnetic Fields and Oscillations, ed. B. Schmieder, A. Hofmann, \& J. Staude, ASP Conf. Ser. 184, 38

Steiner, O. 2003, in NATO Advanced Research Workshop: Turbulence, waves, and instabilities in the solar plasma, ed. R. Erdelyi, \& K. Petrovay, 181

Stenflo, J. O., Solanki, S., Harvey, J. W., \& Brault, J. W. 1984, A\&A, 131, 333

Suematsu, Y. 1990, in Progress of Seismology of the Sun and Stars, ed. Y. Osaki, \& H. Shibahashi, LNP, 367, 211

von der Lühe, O., Soltau, D., Berkefeld, T., \& Schelenz, T. 2003, in Innovative Telescopes and Instrumentation for Solar Astrophysics, ed. S. L. Keil, \& S. V. Avakyan, Proc. SPIE, 4853, 187

Wallace, L., Hinkle, K., \& Livingston, W. 2000, An atlas of sunspot umbral spectra in the visible, from 15000 to $25500 \mathrm{~cm}(-1)(3920$ to 6664 [ $\AA$ ] ) (NOAO)

Wedemeyer-Böhm, S., Kamp, I., Bruls, J., \& Freytag, B. 2005, A\&A, 438, 1043 